\title{
COSMOLOGY, BLACK HOLES AND SHOCK WAVES BEYOND THE HUBBLE LENGTH *
}

\author{
JOEL SMOLLER ${ }^{\dagger}$ AND BLAKE TEMPLE ${ }^{\ddagger}$
}

\begin{abstract}
We construct exact, entropy satisfying shock wave solutions of the Einstein equations for a perfect fluid which extend the Oppeheimer-Snyder (OS) model to the case of non-zero pressure, inside the Black Hole. These solutions put forth a new Cosmological Model in which the expanding Friedmann-Robertson-Walker (FRW) universe emerges from the Big Bang with a shock wave at the leading edge of the expansion, analogous to a classical shock wave explosion. This explosion is large enough to account for the enormous scale on which the galaxies and the background radiation appear uniform. In these models, the shock wave must lie beyond one Hubble length from the FRW center, this threshhold being the boundary across which the bounded mass lies inside its own Schwarzshild radius, $2 M / r>1$, and in this sense the shock wave solution evolves inside a Black Hole. The entropy condition, which breaks the time symmetry by selecting the explosion over the implosion, also implies that the shock wave must weaken until it eventually settles down to a zero pressure OS interface, bounding a finite total mass, that emerges from the White Hole event horizon of an ambient Schwarzschild spacetime. However, unlike shock matching outside a Black Hole, the equation of state $p=\frac{c^{2}}{3} \rho$, the equation of state at the earliest stage of Big Bang physics, is distinguished at the instant of the Big Bang - for this equation of state alone, the shock wave emerges from the Big Bang at a finite nonzero speed, the speed of light, decelerating to a subluminous wave from that time onward. These shock wave solutions indicate a new cosmological model in which the Big Bang arises from a localized White Hole explosion occurring inside a matter filled universe that eventually evolves outward through the White Hole event horizon of an asymptotically flat Schwarzschild spacetime.
\end{abstract}

1. Introduction. In the standard model of cosmology based on a critically expanding, $(k=0)$, Friedmann-Robertson-Walker (FRW) metric, the universe is infinite at each instant after the Big Bang, [11, 21, 24]. The Hubble constant, which measures the recessional velocity of the galaxies, applies to the entire flat space $\mathbf{R}^{3}$-that is, it applies to an entire universe of infinite mass and extent - at each fixed positive time in the standard model. In this paper we present a new cosmological model in which the expansion of the galaxies is a bounded expansion of finite total mass, and the Hubble law applies only to a bounded region of spacetime, (c.f. [21]). If the observed expansion of the galaxies actually only applies to a localized region of spacetime, then it follows that there must be a wave at the leading edge of the expansion. Thus to replace the assumption in the standard model that the universe is infinite at each instant after the Big Bang, we are led to models in which the expansion emerges from an event that is more similar to a classical shock wave explosion, than it is to the usual scenario of the Big Bang.

In previous work, we constructed such models by matching FRW metrics to standard Tolman-Oppenheimer-Volkoff (TOV) metrics, (the metric for a static fluid sphere in general relativity), across a shock wave interface, $[14,21]$. In that work we derived an upper limit on the distance that a shock wave could be from the FRW center, and this distance turned out to be closer than astronomical observations suggest it could be. (Astronomical observations currently extend out to approximately 1.5 Hubble lengths, where all distances are measured at fixed time in the FRW spacetime.) In

\footnotetext{
*Received April 24, 2004; accepted for publication May 19, 2004.

${ }^{\dagger}$ Department of Mathematics, University of Michigan, Ann Arbor, MI 48109, USA (smoller@umich.edu). Supported in part by NSF Applied Mathematics Grant Number DMS-0103998, and by the Institute of Theoretical Dynamics, UC-Davis.

$\ddagger$ Department of Mathematics, University of California, Davis, Davis CA 95616, USA (temple@math.ucdavis.edu). Supported in part by NSF Applied Mathematics Grant Number DMS010-2493, and by the Institute of Theoretical Dynamics, UC-Davis.
} 
this paper we begin by showing that this upper bound on the shock position that we identified in [21] for standard FRW-TOV shock matching, is exactly equal to one Hubble length. Indeed, we show that in order for the shock position to lie beyond one Hubble length in an FRW metric, it is necessary that the spacetime beyond the shock wave lie inside a Black Hole. Thus our previous shock matching limit of one Hubble length results from the fact that a standard TOV metric cannot be continued into a Black Hole, except in the special case when the pressure is zero, (we proved this in [15]). With this motivation, we here derive a new class of gravitational metrics that we call the TOV metric inside the Black Hole. (In contrast to the standard TOV metric, the TOV metric inside the Black Hole is dynamical.) Based on this, we develop a theory of FRW-TOV shock matching inside the Black Hole, and we use this to construct a new class of exact shock wave solutions of the Einstein equations, in which a shock wave is incorporated into the FRW metric at distances arbitrarily far beyond the Hubble length. This demonstrates that the limit in our previous work [21], that the shock position must lie within one Hubble length of the FRW center in FRW-TOV shock matching, can be overcome. In fact, because the mass $M$ of the the TOV metric is constant at each fixed time inside the Black Hole, the TOV metric represents the simplest metric that can incorporate a finite mass cut off of the FRW metric beyond one Hubble length.

In the exact solutions constructed in this paper, the expanding FRW universe emerges behind a subluminous blast wave that explodes outward from the FRW origin $\bar{r}=0$ at the instant of the Big Bang $t=0,{ }^{3}$ at a distance beyond one Hubble length ${ }^{4}$. The distance of one Hubble length is critical in the FRW metric because the total FRW mass $M$ inside radius $\bar{r}$ at fixed time satisfies $\frac{2 M}{\bar{r}}<1$, out to exactly one Hubble length. Thus, one Hubble length marks the event horizon of a Black Hole in a shock wave model in which the mass $M$ is isolated in an asymptotically Schwarzschild metric- the TOV metric in our model. (In contrast to the TOV metric outside the Black Hole, the TOV metric inside the Black Hole can be continued into an event horizon, c.f. the remarks after Theorem 6 below.) After the Big Bang, the shock wave in our exact solution continues to weaken as it expands outward, satisfying the entropy condition for shocks all the way out until the Hubble length eventually catches up to the shock wave ${ }^{5}$. At this instant the shock wave lies at the critical distance of exactly one Hubble length from the FRW center. From this time onward, the shock wave can be approximated by a zero pressure, $k=0$ Oppenheimer-Snyder (OS) interface that emerges from the White Hole event horizon of an ambient Schwarzschild metric of

\footnotetext{
${ }^{3}$ Here, $\bar{r}=R(t) r$ measures radial arclength distance at each fixed time $t$ in FRW coordinates, where $R(t)$ denotes the cosmological scale factor, and $r$ is the standard FRW radial coordinate, c.f. (2.4), (2.5) below.

${ }^{4}$ The Hubble length $c / H(t)=\left[\frac{\dot{R}(t)}{c R(t)}\right]^{-1}$ depends on the cosmological scale factor $R(t)$, and an easy calculation using the Einstein equations shows that in the FRW spacetime, the Hubble length increases with time. Thus more and more galaxies pass inside of the threshold distance of one Hubble length and come into view as time evolves. The Hubble length $c / H_{0}$ at present time is estimated to be on the order of $10^{10}$ light years.

${ }^{5}$ In our cosmological interpretation of the FRW metric, we (loosely) identify the motion of the galaxies with the motion of the FRW fluid, which is taken to be a perfect fluid with nonzero pressure, co-moving with the FRW metric. We show below that, although the shock wave moves outward through the galaxies, $(\dot{r}>0)$, and the Hubble length increases with time, the number of Hubble lengths from the FRW center to the shock wave, (c.f. (5.36) below), as well as the total mass behind the shock wave, both decrease in time in these exact solutions, tending to infinity in backwards time at the instant of the Big Bang. This is no contradiction because the FRW pressure $p$ is assumed nonzero, c.f. Corollary 1 below.
} 
finite mass. (The entropy condition implies that the TOV density and pressure tend to zero as the shock interface approaches the critical distance of one Hubble length.) Thereafter the interface continues out to infinity along a geodesic of the Schwarzschild metric outside the Black Hole. Thus the OS solution gives the large time asymptotics of this new class of shock wave solutions that evolve inside a Black Hole.

One of the surprises in the analysis is that the equation of state that applies at the earliest stage of Big Bang physics, $p=\frac{c^{2}}{3} \rho$, is distinguished by the equations, and only for this equation of state does the shock wave emerge from the Big Bang at a finite nonzero speed, the speed of light, decelerating to a subluminous wave from that time onward.

These new shock wave solutions of the Einstein equations confirm the mathematical consistency of an FRW universe of finite extent and non-zero pressure expanding outward from behind an entropy satisfying shock wave emerging from the origin at subluminous speed beyond one Hubble length at the instant of the Big Bang, a prerequisite for early Big Bang physics. Since the shock wave emerges from the Big Bang beyond one Hubble length, it would account for the thermalization of radiation in a region that is initially well beyond the light cone of an observer positioned at the FRW center. Thus our attempt to incorporate a shock wave beyond one Hubble length has led to unexpected and interesting connections between Big Bang Cosmology and Black Holes. But furthermore, we suggest that general relativity pretty much forces the qualitative behavior we see here into any reasonable model that relaxes the assumption in the standard model that the expansion of the galaxies is of infinite extent at each fixed time. (One could say that in these new models, the Copernican Principle is replaced by the principle in physics that Nothing Is Infinite.)

In Section 2 we transform the FRW metric to standard Schwarzschild coordinates, and use this to discuss the connection between the Hubble length and the Schwarzschild radius. In Section 3 we construct the extension of the zero pressure, $k=0$, OS solution to the interior of a Black Hole by using Eddington-Finkelstein coordinates to regularize the event horizon of the Schwarzschild metric, $[9,17]$. We return to these OS solutions in Section 6 where we argue that the shock wave solutions constructed there continue naturally to the OS solution after the solution has relaxed to almost zero pressure. Thus the OS solutions give the large time asymptotics of our $p \neq 0$ shock wave solutions inside the Black Hole.

In Section 4 we construct the TOV metric inside the Black Hole. We refer to this metric as TOV because the metric components depend only on the radial coordinate $\bar{r}$, but, as in the Schwarzschild metric, the TOV radial coordinate is timelike inside the Black Hole. It follows that the mass function $M$ is constant in each spacelike slice $\bar{r}=$ const. of the TOV metric inside the Black Hole.

In Section 5 we develop the theory of shock matching between a $k=0$ FRW metric and TOV metrics inside the Black Hole. The shock matching techniques introduced in [13] must be modified inside the Black Hole because the conservation constraint used in [13] introduces unphysical solutions. (These solutions are everywhere characteristic relative to the linear partial differential equation for the integrating factor that determines the matching of the metrics.) The analysis leads to the derivation of a system of differential equations that simultaneously describe the time evolution of the shock position together with the outer TOV pressure, and for solutions of these equations, the shock interface must lie beyond one Hubble length from the center in the FRW metric. One interesting feature of the matching is that the radial coordi- 
nate $\bar{r}$, a timelike coordinate in the TOV metric, is identified through shock matching with the FRW spacelike coordinate $\bar{r}$ that measures radial arclength distance from the center at each fixed value of the (standard) time $t$ in the FRW metric. Another interesting feature is that the mass function $M$, which is continuous across shocks as a consequence of shock matching, has the physical interpretation as a total mass inside radius $\bar{r}$ in the FRW metric, but $M$ has no such interpretation in the TOV metric by itself, and in fact we know of no general physical interpretation of the mass function $M$ inside the Black Hole.

In Section 6 we formulate the entropy condition, and construct a class of global, entropy satisfying shock wave solutions of the Einstein equations under the simplifying assumption that the FRW sound speed $\sqrt{\sigma}$ is constant, (that is, we assume the FRW equation of state $p=\sigma \rho, \sigma=$ const. $>0$, c.f. [14]). This includes the important case $\sigma=\frac{c^{2}}{3}$, usually assumed at the earliest stage of Big Bang physics. (This is the equation of state in the extreme relativistic limit of free particles, and for pure radiation, [13].) Remarkably, under a change of variables, the shock matching equations of Section 5 reduce to a planar autonomous system when $\sigma=$ const., and this system is amenable to global analysis, a requirement for the construction of solutions in the large. It is very interesting that the special value $\sigma=c^{2} / 3$ plays a distinguished role, and at this unique value, the shock wave is everywhere subluminous after the Big Bang, but emerges from the Big Bang at exactly the speed of light. For all other values of $\sigma$, we prove that the shock speed at the instant of the Big Bang is either zero, or infinite, and is everywhere subluminous if and only if $\sigma \leq c^{2} / 3$. This is surprising because the equation of state $p=\frac{c^{2}}{3} \rho$ played no special role in shock matching outside the Black Hole.

The class of exact solutions in Section 6 describes the global dynamics of an FRW universe of finite extent which explodes outward behind an entropy satisfying, subluminous shock wave, emerging from the origin, beyond one Hubble length, at the instant of the Big Bang. Because the TOV metric inside the Black Hole has nonzero density and pressure, it follows that, unlike the OS solutions of Section 3, these new exact shock wave solutions do not require any part of the empty space Schwarzschild solution inside the Black Hole for their construction. On the other hand, the fact that the OS solution gives the qualitative large time behavior of the solutions independent of $\sigma$, (a consequence of the entropy condition alone), suggests that the qualitative features of these solutions may be generic for a large class of equations of state.

In Section 7 we obtain estimates for the shock position. The conclusion of Theorem 9 is this: Let $t_{0}$ be the first time at which the shock becomes visible at the FRW center. In our exact solutions, we assume the FRW equation of state $p=\sigma \rho$, $\sigma=$ const, $0<\sigma \leq 1 / 3$. (We use the convention that we take $c=1$ when convenient.) For these solutions, the shock wave will first become visible at the center $\bar{r}=0$ of the FRW spacetime at FRW time $t=t_{0}$, at the moment when the Hubble length satisfies

$$
\frac{1}{H\left(t_{0}\right)}=\frac{1+3 \sigma}{2} r_{*}
$$

where $r_{*}$ is the FRW position of the shock at the instant of the Big Bang. (The fact that $r_{*}>0$ at the Big Bang does not imply that we are artificially placing the shock wave away from the center at the Big Bang because $r$ is singular relative to arclength $\bar{r}=R r$ when $R=0$. ) At this time, the number of Hubble lengths $\sqrt{N}_{0}$ from the 
FRW center to the shock wave at time $t=t_{0}$ satisfies the bounds

$$
1 \leq \frac{2}{1+3 \sigma} \leq \sqrt{N}_{0} \leq \frac{2}{1+3 \sigma} e^{\sqrt{3 \sigma}\left(\frac{1+3 \sigma}{1+\sigma}\right)} .
$$

Thus, in particular, the shock wave will still lie beyond one Hubble length at the FRW time $t_{0}$ when it first becomes visible. Furthermore, the time $t_{c r i t}>t_{0}$ at which the shock wave will emerge from the White Hole event horizon, given that $t_{0}$ is the first instant at which the shock becomes visible at the FRW center, can be estimated by

$$
\frac{2}{1+3 \sigma} e^{\frac{1}{4} \sigma} \leq \frac{t_{\text {crit }}}{t_{0}} \leq \frac{2}{1+3 \sigma} e^{\frac{2 \sqrt{3 \sigma}}{1+\sigma}}
$$

and by the better estimate

$$
e^{\frac{\sqrt{6}}{4}} \leq \frac{t_{c r i t}}{t_{0}} \leq e^{\frac{3}{2}}
$$

in the case $\sigma=1 / 3$. For example, these inequalities imply that in the OS limit $\sigma=0$,

$$
\sqrt{N_{0}}=2, \quad \frac{t_{c r i t}}{t_{0}}=2
$$

and in the limit $\sigma=1 / 3$,

$$
1 \leq \sqrt{N_{0}} \leq 4.5, \quad 1.8 \leq \frac{t_{c r i t}}{t_{0}} \leq 4.5 .
$$

We conclude in these shock wave cosmological models, that at the moment $t_{0}$ when the shock wave first becomes visible at the FRW center, it must lie within 4.5 Hubble lengths of the center. Throughout the expansion up until this time, the expanding universe must lie entirely within a Black Hole - the universe will eventually emerge from this Black Hole, but not until some later time $t_{c r i t}$, where $t_{c r i t}$ does not exceed $4.5 t_{0}$.

Concluding remarks are made in the final section.

2. The Hubble Distance and the Schwarzschild Radius. According to Einstein's Theory of General Relativity, all properties of the gravitational field are determined by a Lorentzian spacetime metric tensor $g$, whose line element in a given coordinate system $x=\left(x^{0}, \ldots, x^{3}\right)$ is given by

$$
d s^{2}=g_{i j} d x^{i} d x^{j} .
$$

(We use the Einstein summation convention whereby all repeated up-down indices are assumed summed from 0 to 3.) The components $g_{i j}$ of the gravitational metric $g$ satisfy the Einstein equations,

$$
G^{i j}=\kappa T^{i j}, \quad T^{i j}=\left(\rho c^{2}+p\right) w^{i} w^{j}+p g^{i j},
$$

where we assume the stress-energy tensor $T$ of a perfect fluid. Here $G$ is the Einstein curvature tensor, 


$$
\kappa=\frac{8 \pi \mathcal{G}}{c^{4}}
$$

is the coupling constant, $\mathcal{G}$ is Newton's gravitational constant, $c$ is the speed of light, $\rho c^{2}$ is the energy density, $p$ is the pressure, and $\mathbf{w}=\left(w^{0}, \ldots, w^{3}\right)$ are the components of the 4-velocity of the fluid, c.f. [24]. We use the convention that we take $c=1$ and $\mathcal{G}=1$ when convenient.

In this section we consider the FRW metric, a spacetime metric whose line element takes the form

$$
d s^{2}=-d t^{2}+R(t)^{2}\left(\frac{d r^{2}}{1-k r^{2}}+r^{2} d \Omega^{2}\right),
$$

where $R(t)$ is the so-called cosmological scale factor, and $d \Omega^{2}=d \theta^{2}+\sin ^{2} \theta d \phi^{2}$ denotes the line element on the unit 2-sphere. The FRW metric describes the time evolution of a three dimensional space of constant scalar curvature, (the $t=$ const. surfaces), and $k$ can be assumed to take one of the values $\{-1,0,1\}$ via a rescaling of the radial coordinate $r$. Radial distance at each fixed time in the FRW metric is measured by $\bar{r}$, where

$$
\bar{r}=R(t) r,
$$

and it is standard to rescale time so that $R$ goes from 0 to 1 , where $R=0, t=0$ corresponds to the Big Bang, and $R=1, t=t_{0}$ corresponds to present time.

The Hubble constant $H$, (which actually depends on $t$ ), is given in terms of $R(t)$ by

$$
H \equiv H(t)=\frac{\dot{R}(t)}{R(t)}
$$

and we let $H_{0}=H\left(t_{0}\right)$ denote the present value of the Hubble constant.

The Hubble length $c / H$, (the reciprocal of the Hubble constant when we take $c=1$ ), gives a length scale determined by the expansion rate of the galaxies, and can be interpreted as the travel distance for a light ray starting at the Big Bang and ending at time $t$. (That is, the age of the universe is approximately $H_{0}^{-1} \approx 10^{10}$ years, so the Hubble length $c / H_{0} \approx 10^{10}$ light years, is a measure of the distance light travels during this time interval, [24].) The Hubble length is thus a measure of the distance to the furthest objects that can be seen in the universe at a given time. (Estimates for this distance in terms of radial arclength $\bar{r}$ at fixed FRW time $t$ are given in Theorems 1 and 2 below.)

Putting the metric ansatz (2.4) into the Einstein equations (2.2) gives the equations for the FRW metric, [24],

$$
H^{2}=\left(\frac{\dot{R}}{R}\right)^{2}=\frac{\kappa}{3} \rho-\frac{k}{R^{2}},
$$

and 


$$
\dot{\rho}=-3(p+\rho) H
$$

The unknowns $R, \rho$ and $p$ are assumed to be functions of the FRW coordinate time $t$ alone, and "dot" denotes differentiation with respect to $t$. In this paper, we focus on the case of critical expansion, the case $k=0,[1,24]$.

When $k=0$, the $t=$ const. surfaces in the FRW metric are infinite, flat, Euclidean 3-space. It follows that the standard model of cosmology based on a critically expanding FRW metric, [1], implicitly assumes that the expansion rate $H$ of the universe is constant all the way out to infinity at each fixed time $t$ in the FRW metric - this even though, based on lookback time, we can in principle only observe the universe out to about $10^{10}$ light years.

If, on the other hand, there is a shock wave at the leading edge of the expansion of the galaxies, then the following question presents itself: What is the critical radius $\bar{r}_{\text {crit }}$ at each fixed time $t$ in a $k=0$ FRW metric such that the total mass inside a shock wave positioned beyond that radius puts the universe inside a Black Hole? Indeed, there must always be such a critical radius because the total mass $M(\bar{r}, t)$ inside radius $\bar{r}$ in the FRW metric at fixed time $t$ increases like $\bar{r}^{3}$, and so at each fixed time $t$ we must have $\bar{r}>2 M(\bar{r}, t)$ for small enough $\bar{r}$, while the reverse inequality holds for large $\bar{r}$. Thus for every time $t$ there must exist a smallest $\bar{r}=\bar{r}_{\text {crit }}$ for which $\bar{r}_{c r i t}=2 M\left(\bar{r}_{\text {crit }}, t\right)$. This critical radius then marks the Schwarzschild radius in the metric that lies beyond the shock wave when it is at that position. We will presently show that when $k=0, \bar{r}_{\text {crit }}$ equals the Hubble length, and this explains the shock matching limit uncovered in our previous work in terms of the Hubble length, (c.f. Section 6, [21]). That is, we cannot match a critically expanding FRW metric to a classical TOV metric beyond one Hubble length without continuing the TOV solution into a Black Hole -and we showed in [15] that the standard TOV metric cannot be continued into a Black Hole.

At this point we make a note on terminology: we say that a radial solution of the Einstein equations is inside the Black Hole provided that $\frac{2 M}{\bar{r}}>1$. If further, the radial coordinate $\bar{r}$ is always increasing along timelike curves, then we also refer to such a region $\frac{2 M}{\bar{r}}>1$ as a White Hole, but we will nevertheless use the term Black Hole whenever $\frac{2 M}{\bar{r}}>1$.. For example, in the Kruskal development of a Schwarzschild Black Hole there are four quadrants, and one quadrant contains the Black Hole singularity and the opposite quadrant, (its time reversal), contains the White Hole singularity, [9]. Both quadrants lie within the region where $\frac{2 M}{\bar{r}}>1$, and the quadrant with the Black [resp. White] Hole singularity at $\bar{r}=0$ has the property that all timelike geodesics end [resp. begin] at the singularity in finite proper time ${ }^{6}$. Thus if $\bar{r}_{c r i t}=c / H$, and the mass function $M$ is continuous across shock waves, (both of which we demonstrate below), then it follows that one can incorporate a shock wave into an FRW metric beyond one Hubble length only if the metric beyond the shock wave lies inside a Black Hole, where $\frac{2 M(\bar{r}, t)}{\bar{r}}>1$.

\footnotetext{
${ }^{6} \mathrm{By}$ itself, Einstein's theory is time reversible, the direction of forward time is left undetermined, and extra conditions like entropy conditions for shocks are needed to determine the direction of forward time. In the region $2 M / \bar{r}>1$ outside the event horizon, the Schwarzschild metric is symmetric under time reversal in the sense that the time reversal of a geodesic remains a geodesic. However in the region $2 M / \bar{r}<1$, the time reversal of the Schwarzschild metric takes the Black Hole region beyond $t=+\infty$ to the White Hole region before $t=-\infty$. Thus without extra conditions, the expanding White Hole is just as admissible a solution of the Einstein equations as is its time reversal, the collapsing Black Hole.
} 
We now verify that $\bar{r}_{c r i t}=c / H$, and that the Hubble length is the limit for FRWTOV shock matching outside a Black Hole, c.f. [21]. To this end, write the FRW metric (2.4) in standard Schwarzschild coordinates $\overline{\mathbf{x}}=(\bar{r}, \bar{t})$ where the metric takes the form

$$
d s^{2}=-B(\bar{r}, \bar{t}) d \bar{t}^{2}+A(\bar{r}, \bar{t})^{-1} d \bar{r}^{2}+\bar{r}^{2} d \Omega^{2},
$$

and the mass function $M(\bar{r}, \bar{t})$ is defined through the relation

$$
A=1-\frac{2 M}{\bar{r}} \text {. }
$$

(We have set $\mathcal{G}=c=1$, and our notation is to denote the standard, non-angular FRW coordinates by $\mathbf{x}=\left(x^{0}, x^{1}\right)=(t, r)$, but TOV coordinates are denoted by $\overline{\mathbf{x}}=\left(\bar{x}^{0}, \bar{x}^{1}\right)=(\bar{r}, \bar{t})$ because we work inside the Black Hole where $A<0$ and $\bar{r}$ is the timelike coordinate.) It is well known that a general spherically symmetric metric can be transformed to the form (2.9) by coordinate transformation, [24]. To obtain $A$ and $B$ for the FRW metric (2.4), set $\bar{r}=R r$, so that

$$
d r=\frac{d \bar{r}}{R}-\frac{\bar{r}}{R^{2}} \dot{R} d t
$$

Using this in the FRW line element we find

$$
d s^{2}=-C d t^{2}+D d \bar{r}^{2}+2 E d \bar{r} d t+\bar{r}^{2} d \Omega^{2},
$$

where

$$
\begin{aligned}
C & =\frac{1-k r^{2}-H^{2} \bar{r}^{2}}{1-k r^{2}}, \\
D & =\frac{1}{1-k r^{2}}, \\
E & =-\frac{H \bar{r}}{1-k r^{2}} .
\end{aligned}
$$

Now define the time coordinate $\bar{t}=\bar{t}(t, \bar{r})$ by

$$
d \bar{t}=(\psi C) d t-(\psi E) d \bar{r},
$$

where $\psi$ is an integrating factor to be determined by the equation

$$
\frac{\partial}{\partial \bar{r}}(\psi C)+\frac{\partial}{\partial t}(\psi E)=0 .
$$

Equation (2.16) implies that $d \bar{t}$ is an exact differential, and the choice of $E$ and $C$ applied to $(2.15)$ removes the cross term from the metric $(2.11)$. In $(\bar{r}, \bar{t})$ coordinates the FRW metric takes the form

$$
d s^{2}=-\frac{1}{C \psi^{2}} d \bar{t}^{2}+\left(D+\frac{E^{2}}{C}\right) d \bar{r}^{2}+\bar{r}^{2} d \Omega^{2}
$$


Using (2.12)-(2.14) in (2.17) gives

$$
d s^{2}=-\frac{1}{\psi^{2}}\left\{\frac{1-k r^{2}}{1-k r^{2}-H^{2} \bar{r}^{2}}\right\} d \bar{t}^{2}+\left\{\frac{1}{1-k r^{2}-H^{2} \bar{r}^{2}}\right\} d \bar{r}^{2}+\bar{r}^{2} d \Omega^{2} .
$$

Comparing (2.18) with (2.9) we obtain

$$
\begin{aligned}
A^{-1} & =\frac{1}{1-k r^{2}-(\bar{r} H)^{2}}, \\
B & =\frac{1}{\psi^{2}}\left(\frac{1-k r^{2}}{1-k r^{2}-(\bar{r} H)^{2}}\right) .
\end{aligned}
$$

Note that the characteristic curves for (2.16) are given by

$$
\frac{d \bar{r}}{d t}=\frac{C}{E}=\frac{-1+k r^{2}+H^{2} \bar{r}^{2}}{H \bar{r}}
$$

the RHS being a smooth function for all $t>0$ for which $H \neq 0 \neq \bar{r}$. (By (2.7), the condition $H=\frac{\dot{R}}{R} \neq 0$ holds for all $t>0$ when $k=0,-1$, and it holds except at the turning point $\dot{R}=0$ in the case when $k=+1$, c.f. [24].) It follows that a solution $\psi$ of (2.16) exists in a neighborhood of any point. In fact, we have more: we can assign arbitrary initial values for $\psi$ on any surface that is non-characteristic for (2.16), and then use these values to solve (2.16) for $\psi$ in a neighborhood of that surface, c.f. $[14,24]$. By (2.20), this implies that we can assign arbitrary (non-negative) values for $B$ on any non-characteristic surface. We conclude that (2.18)-(2.20) gives the FRW metric in standard Schwarzschild form, the coefficient of $d \bar{r}^{2}$ is determined independently of $\psi$, and values of $B$ can be freely assigned (locally) on any smooth non-characteristic surface in $(t, \bar{r})$ space, thus determining initial values for equation (2.16).

Moreover, using (2.19) in (2.10), it follows that

$$
\begin{aligned}
M(t, \bar{r}) & =\frac{\bar{r}}{2}(1-A)=\frac{\bar{r}}{2}\left(\bar{r}^{2} H^{2}\right)=\frac{1}{3} \frac{\kappa}{2} \rho \bar{r}^{3} \\
& =\frac{\kappa}{2} \int_{0}^{\bar{r}} \rho(t) s^{2} d s .
\end{aligned}
$$

Since in the FRW metric $\bar{r}=R r$ measures arclength along radial geodesics at fixed time, we see from (2.22) that $M(t, \bar{r})$ has the physical interpretation as the total mass inside radius $\bar{r}$ at time $t$ in the FRW metric.

From here on, we restrict to the case of critical expansion, the case $k=0$. In this case, since $\frac{2 M}{\bar{r}}=1-A$, one sees from (2.19) that $\bar{r}=H^{-1}$ is equivalent to $\frac{2 M}{\bar{r}}=1$, and so the following equivalences are valid at any fixed time $t$ :

$$
\bar{r}=H^{-1} \quad \text { iff } \quad \frac{2 M}{\bar{r}}=1 \text { iff } A=0 .
$$

We conclude that $\bar{r}=H^{-1}$ is the critical length scale for the FRW metric at fixed time $t$ in the sense that $\frac{2 M}{\bar{r}}-1$ changes sign at $\bar{r}=H^{-1}$, and so the universe lies inside a Black Hole beyond $\bar{r}=H^{-1}$, as claimed above. 
From the FRW equation (2.7), it also follows that $\bar{r}=H^{-1}$ iff

$$
r^{2}=\frac{3}{\kappa} \frac{1}{\rho R^{2}} .
$$

This explains the condition

$$
r^{2} \leq \frac{3}{\kappa} \frac{1}{\rho R^{2}}
$$

given in equation (6.2) of [21] for the maximal position of a shock wave in FRW-TOV matching. This condition was derived in [21] from a different point of view. Thus (2.23), (2.24) provides a physical interpretation for the bound (6.2) in [21]; namely, that the shock wave lies inside the Hubble length.

The Hubble length $\bar{r}_{c r i t}=\frac{c}{H}$ is also the critical distance at which the outward expansion of the FRW metric exactly cancels the inward advance of a radial light ray impinging on an observer positioned at the origin of a $k=0$ FRW metric. Indeed, by (2.4), a light ray traveling radially inward toward the center of an FRW coordinate system satisfies,

$$
c^{2} d t^{2}=R^{2} d r^{2}
$$

so that

$$
\frac{d \bar{r}}{d t}=\dot{R} r+R \dot{r}=H \bar{r}-c=H\left(\bar{r}-\frac{c}{H}\right)>0,
$$

if and only if

$$
\bar{r}>\frac{c}{H} .
$$

Thus the arclength distance from the origin to an inward moving light ray at fixed time $t$ in a $k=0$ FRW metric will actually increase as long as the light ray lies beyond the Hubble length. An inward moving light ray will, however, eventually cross the Hubble length and reach the origin in finite proper time, due to the increase in the Hubble length with time.

We now calculate the infinite redshift limit in terms of the Hubble length. It is well known that light emitted at $\left(t_{e}, r_{e}\right)$ at wavelength $\lambda_{e}$ in an FRW spacetime will be observed at $\left(t_{0}, r_{0}\right)$ at wavelength $\lambda_{0}$ if

$$
\frac{R_{0}}{R_{e}}=\frac{\lambda_{0}}{\lambda_{e}}
$$

Moreover, the redshift factor $z$ is defined by

$$
z=\frac{\lambda_{0}}{\lambda_{e}}-1 .
$$

Thus, infinite redshifting occurs in the limit $R_{e} \rightarrow 0$, where $R=0, t=0$ is the Big Bang. 
Consider now a light ray emitted at the instant of the Big Bang, and observed at the FRW origin at present time $t=t_{0}$. Let $r_{\infty}$ denote the FRW coordinate at time $t \rightarrow 0$ of the furthestmost objects that can be observed at the FRW origin before time $t=t_{0}$. Then $r_{\infty}$ marks the position of objects at time $t=0$ whose radiation would be observed as infinitly redshifted, (assuming no scattering). Note then that a shock wave emanating from $\bar{r}=0$ at the instant of the Big Bang, will be observed at the FRW origin before present time $t=t_{0}$ only if its position $r$ at the instant of the Big Bang satisfies $r<r_{\infty}$. We now estimate $r_{\infty}$.

First, from (2.26) it follows that an incoming radial light ray in an FRW metric follows a lightlike trajectory $r=r(t)$ if

$$
r-r_{e}=-\int_{t_{e}}^{t} \frac{d \tau}{R(\tau)}
$$

and thus

$$
r_{\infty}=\int_{0}^{t_{0}} \frac{d \tau}{R(\tau)}
$$

We now prove the following theorem:

THEOREM 1. If the pressure $p$ satisfies the bounds

$$
0 \leq p \leq \frac{1}{3} \rho,
$$

then for any equation of state, the age of the universe $t_{0}$ and the infinite red shift limit $r_{\infty}$ are bounded in terms of the Hubble length by

$$
\begin{gathered}
\frac{1}{2 H_{0}} \leq t_{0} \leq \frac{2}{3 H_{0}}, \\
\frac{1}{H_{0}} \leq r_{\infty} \leq \frac{2}{H_{0}} .
\end{gathered}
$$

(We have assumed that $R=0$ when $t=0$ and $R=1$ when $t=t_{0}$ and $H=H_{0}$. Note that $p=\frac{1}{3} \rho$ is the extreme relativistic limit of free particles, as well as the equation of state for pure radiation, [24].)

Proof. Integrating (2.7) we obtain

$$
R(t)=e^{-\int_{t}^{t_{0}} \sqrt{\frac{\kappa \rho}{3}} d t},
$$

and, from (2.8) we have

$$
-\int_{\infty}^{\rho} \frac{d \rho}{(p+\rho) \sqrt{\rho}}=\sqrt{3 \kappa} t
$$


Thus, if we know $p$ as a function of $\rho$, then we can integrate (2.33) to obtain $\rho$ as a function of $t$, and then use this in (2.32), which can then be used in (2.28) to calculate $r_{\infty}$. Assuming (2.29), we can estimate (2.33) by

$$
-\int_{\infty}^{\rho} \frac{d \rho}{\frac{4}{3} \rho^{3 / 2}} \leq \sqrt{3 \kappa} t \leq-\int_{\infty}^{\rho} \frac{d \rho}{\rho^{3 / 2}}
$$

which leads to

$$
\frac{3}{4 \kappa t^{2}} \leq \rho \leq \frac{4}{3 \kappa t^{2}} .
$$

Since (2.7) is $H^{2}=\frac{\kappa}{3} \rho$ when $k=0,(2.34)$ gives

$$
\frac{1}{2 t} \leq H \leq \frac{2}{3 t}
$$

which implies (2.30). To estimate $R(t)$, write $(2.35)$ in the form

$$
\frac{1}{2 t} \leq \frac{d}{d t} \ln R \leq \frac{2}{3 t}
$$

which integrates to

$$
\left(\frac{t}{t_{0}}\right)^{\frac{2}{3}} \leq R \leq\left(\frac{t}{t_{0}}\right)^{\frac{1}{2}}
$$

Finally, using (2.36) in (2.28) gives

$$
\int_{0}^{t_{0}}\left(\frac{t_{0}}{t}\right)^{1 / 2} d t \leq r_{\infty} \leq \int_{0}^{t_{0}}\left(\frac{t_{0}}{t}\right)^{2 / 3} d t
$$

which leads directly to $(2.31)$.

The next theorem gives closed form solutions of the FRW equations (2.7), (2.8) in the case when the sound speed $\sqrt{\sigma} \equiv$ constant. These solutions are the starting point of the exact shock wave solutions constructed in Section 6 . As a special case we recover the bounds in (2.30) and (2.31) from the cases $\sigma=0$ and $1 / 3$.

Theorem 2. Assume $k=0$ and the equation of state

$$
p=\sigma \rho,
$$

where $\sigma$ is assumed constant,

$$
0 \leq \sigma \leq 1
$$


Then, (assuming an expanding universe $\dot{R}>0$ ), the solution of system (2.7), (2.8) satisfying $R=0$ at $t=0$ and $R=1$ at $t=t_{0}$ is given by,

$$
\begin{gathered}
\rho=\frac{4}{3 \kappa(1+\sigma)^{2}} \frac{1}{t^{2}}, \\
R=\left(\frac{t}{t_{0}}\right)^{\frac{2}{3(1+\sigma)}}, \\
\frac{H}{H_{0}}=\frac{t_{0}}{t} .
\end{gathered}
$$

Moreover, the age of the universe $t_{0}$ and the infinite red shift limit $r_{\infty}$ are given exactly in terms of the Hubble length by

$$
\begin{aligned}
t_{0} & =\frac{2}{3(1+\sigma)} \frac{1}{H_{0}}, \\
r_{\infty} & =\frac{2}{1+3 \sigma} \frac{1}{H_{0}} .
\end{aligned}
$$

From (2.41) we conclude that a shock wave will be observed at the FRW origin before present time $t=t_{0}$ only if its position $r$ at the instant of the Big Bang satisfies $r<\frac{2}{1+3 \sigma} \frac{1}{H_{0}}$. Note that $r_{\infty}$ ranges from one half to two Hubble lengths as $\sigma$ ranges from 1 to 0 , taking the intermediate value of one Hubble length at $\sigma=1 / 3$, c.f. (2.31).

Proof. Formulas (2.37)-(2.39) follow directly from (2.32)-(2.33), and agree with the formulas given in [14]. Differentiating (2.38) at $t=t_{0}$ gives (2.40), and using (2.38) and (2.40) in (2.28) gives (2.41).

CoROllary 1. If $p=\sigma \rho, \sigma=$ const. $>0$, then the total mass inside radius $r=$ const, (that is, inside a ball whose boundary is comoving with the galaxies), decreases in time.

Proof. Using (2.37)-(2.38) in (2.22), it follows that

$$
M=\frac{\kappa}{2} \int_{0}^{\bar{r}} \rho(t) s^{2} d s=\frac{2 \bar{r}^{3}}{9(1+\sigma)^{2} t_{0}^{\frac{2}{1+\sigma}}} t^{\frac{-2 \sigma}{1+\sigma}}
$$

so $\dot{M}<0$ if $\sigma>0$,

3. The OS Solution Inside the Black Hole. The simplest model of a localized FRW metric contained within a shock boundary that lies beyond the Hubble distance, is one in which $p \equiv 0$, and the FRW metric is matched to the Schwarzschild metric at a contact discontinuity interface positioned inside the Black Hole of the Schwarzschild metric. This poses the problem of extending the OS solution smoothly into the interior of a Schwarzschild Black Hole. In this section we construct the OS solution inside the Black Hole in the case $k=0$, making the point of discussing it from the FRW point of view, in terms of the Hubble length. (See [10] and [9] for a discussion of the case $k<0$.) The $k=0$ OS solution inside the Black Hole will give 
us a point of comparison for the $p \neq 0$ shock wave models constructed in Sections 5 and 6 , and we argue in Section 7 that the (expanding) OS solution also describes the large time asymptotics of these shock wave models. When $p \neq 0$, the resulting interface is a shock wave, and an entropy condition breaks the time symmetry. In our examples of Section 6, it is the outward expanding solution (explosion) that globally meets the entropy condition, (not the inward collapse), thus making the cosmological interpretation of the model relevant. In contrast, the interface in the OS solution is time reversible, but the collapsing solution is the one that is relevant in the standard interpretation of the OS solution as a pressureless sphere collapsing into a Black Hole, $[10,9]$.

Our method is to match the $k=0$ FRW metric to the empty space Schwarzschild metric written in Eddington-Finkelstein (EF) coordinates [9], across a shock interface ${ }^{7}$ that lies beyond the Hubble length on the FRW side of the shock. As in a classical explosion, we assume that the FRW metric lies inside a bounded region behind an outgoing shock interface, and for the OS solution we assume that the Schwarzschild metric describes the spacetime beyond the interface. Thus in the OS model, the shock wave (contact discontinuity) marks the leading edge of the expansion of the FRW metric.

The EF coordinates provide a regularization of the Schwarzschild metric at the event horizon, and the mapping from the EF spacetime to the Schwarzschild spacetime is a 1-1 mapping, [9]. Both coordinate systems cover the region of the Schwarzschild metric outside the event horizon, together with that portion of the Black Hole that is coordinatized by the Schwarzschild coordinates. Here, since we are dealing with an expanding FRW solution, we work with the time reversal of these metrics, which covers the region beyond the event horizon, together with the White Hole region inside the Black Hole We show that with this latter time orientation, we can match the EF metric to the spacetime described by a critically expanding FRW metric across a discontinuity at finite radius, and its time reversal provides the corresponding matching to the critically contracting FRW metric.

We return to these OS solutions in Section 6 where we argue that the shock wave solutions constructed there continue naturally to the OS solution after the TOV density and pressure tend to zero, assuming that the shock has relaxed to a sufficiently weak wave. But we find this OS model interesting in its own right because it not only provides the simplest model in which the expansion of the galaxies corresponds to the expansion of a finite total mass with a wave at the leading edge of the expansion, but it also embeds the Big Bang singularity of an FRW metric within the singularity of a larger spacetime - the larger spacetime being the empty space Schwarzschild solution, and its singularity being the White Hole singularity that lies inside the event horizon in the Kruskal development of the Schwarzschild metric. Such an embedding is possible only under the assumption that the Hubble law applies to a bounded region of spacetime, because the infinite FRW metric cannot be matched to the Schwarzschild metric.

In EF coordinates, the Schwarzschild line element takes the form

$$
d \hat{s}^{2}=-A d \hat{t}^{2}-2 d \bar{r} d \hat{t}+\bar{r}^{2} d \Omega^{2}
$$

where

\footnotetext{
${ }^{7}$ The interface in the OS solutions is, in the language of gas dynamics, a contact discontinuity, which is time reversible because neither mass nor momentum cross the interface.
} 


$$
A=1-\frac{2 M}{\bar{r}},
$$

and $M$ is the constant mass of the Schwarzschild solution. Indeed, using (2.11)-(2.16), the time coordinate $\bar{t}$ that eliminates the mixed term in (3.1) satisfies

$$
d \bar{t}=\psi A d \hat{t}-\psi \bar{r}
$$

where $\psi$ satisfies

$$
\frac{\partial}{\partial \hat{t}} \psi+\frac{\partial}{\partial \bar{r}}(\psi A)=0 .
$$

The solution to $(3.3),(3.4)$ is

$$
\begin{gathered}
\psi=1 / A, \\
\bar{t}=t-\bar{r}-\ln |\bar{r}-2 G M|,
\end{gathered}
$$

and in $(\bar{r}, \bar{t})$-coordinates the metric takes the standard Schwarzschild form

$$
d s^{2}=-A d \vec{t}^{2}+\frac{1}{A} d \bar{r}^{2}+\bar{r}^{2} d \Omega^{2}
$$

Thus (3.6) defines a $1-1$ mapping $(\hat{t}, \bar{r}) \rightarrow(\bar{r}, \bar{t})$ taking $\{\bar{r}>2 M\} \times \mathbf{R}$ to itself, and another $1-1$ mapping $(\hat{t}, \bar{r}) \rightarrow(\bar{r}, \bar{t})$ taking $\{0<\bar{r}<2 M\} \times \mathbf{R}$ to itself. This verifies the claim that the EF metric covers two of the four quadrants of the (maximal) Kruskal development of the Schwarzschild metric in a 1-1 fashion. Unlike the Schwarzschild metric, the EF metric is smooth at $\bar{r}=2 M$, and this is reflected in the singularity of the transformation (3.6) at $\bar{r}=2 M$. Note that in $(\bar{r}, \bar{t})$ coordinates, the vector field $\frac{\partial}{\partial \bar{r}}$ is timelike, spacelike, when $\bar{r}<2 M, \bar{r}>2 M$, respectively; while in $(\hat{t}, \bar{r})$ coordinates, the vector field $\frac{\partial}{\partial \bar{r}}$ is lightlike. This is no contradiction because $\frac{\partial}{\partial \bar{r}}$ points along the level curves of its complementary coordinates in a given coordinate system. On the other hand, the vector fields $\frac{\partial}{\partial t}$ and $\frac{\partial}{\partial t}$ both point along the level curves of $\bar{r}$, and hence retain the same character as spacelike or timelike according to the sign of $A$.

The $k=0$ FRW metric is given in (2.4) as

$$
d s^{2}=-d t^{2}+R(t)^{2} d r^{2}+R(t)^{2} r^{2} d \Omega^{2},
$$

where $R(t)$ is the cosmological scale factor. To obtain a matching of the two metrics (3.1), (3.8), that is smooth at the Schwarzschild radius, we match them in $(\hat{t}, \bar{r})$ coordinates. To start, first match the spheres of symmetry by setting, (c.f. [13, 24]),

$$
\bar{r}=R r .
$$


Writing $(2.4)$ in $(t, \bar{r})$ coordinates yields the following form of the FRW metric derived in (2.11)-(2.14) above upon setting $k=0$ :

$$
d s^{2}=-d t^{2}+\left\{d \bar{r}^{2}-2 H \bar{r} d \bar{r} d t+\frac{\dot{R}^{2}}{R^{2}} \bar{r}^{2} d t^{2}\right\}+\bar{r}^{2} d \Omega^{2}
$$

We now construct the transformation $\hat{t}=\hat{t}(t, \bar{r})$ by finding functions $b(t, \bar{r})$ and $\phi(t, \bar{r})$ such that $b$ and $\phi$ satisfy

$$
d \hat{t}=\phi d t+\phi b d \bar{r}
$$

and

$$
\phi_{\bar{r}}-(\phi b)_{t}=0 .
$$

Equation (3.11) implies that $d \hat{t}$ is an exact differential, and thus (3.10) defines the coordinate transformation for $\hat{t}$ as a function of $t$ and $\bar{r}$. In order to find $\phi$ and $b$, we start from (3.10) and write

$$
d t=\frac{1}{\phi}\{d \hat{t}-\phi b d \bar{r}\}
$$

and so

$$
d t^{2}=\frac{1}{\phi^{2}}\left\{d \hat{t}^{2}-2 b \phi d \hat{t} d \bar{r}+b^{2} \phi^{2} d \bar{r}^{2}\right\} .
$$

Using these in (3.9) we obtain

$$
\begin{gathered}
d s^{2}=\frac{1}{\phi^{2}}\left(\frac{\dot{R}^{2}}{R^{2}} \bar{r}^{2}-1\right) d \hat{t}^{2}+\frac{2}{\phi}\left\{-b\left(-1+\frac{\dot{R}^{2}}{R^{2}} \bar{r}^{2}\right)-H \bar{r}\right\} d \hat{t} d \bar{r}+ \\
\left\{1+b\left[b\left(-1+\frac{\dot{R}^{2}}{R^{2}} \bar{r}^{2}\right)+\frac{2 \dot{R}}{R} \bar{r}\right]\right\} d \bar{r}^{2}
\end{gathered}
$$

The outline of our procedure for matching the components of the metric (3.14) to the components of the metric (3.1) is as follows: We first set the coefficient of $d \bar{r}^{2}$ equal to zero in (3.14) and solve for the function $b$. We then match the coefficients of the cross terms $d \hat{t} d \bar{r}$ by setting the $d \hat{t} d \bar{r}$ coefficient in (3.14) equal to -2 and then solve for the value of $\phi$ on the shock surface. Using the values of $b$ and $\phi$ in the matching of the $d \hat{t}^{2}$ coefficients, we obtain a formula for the shock surface. Finally, we show that the shock surface is non-characteristic for the PDE (3.11), implying that we can use the initial condition for $\phi$ to solve for $\phi$ in a neighborhood of the shock surface, and thereby determine a nonsingular coordinate $\bar{t}=\bar{t}(t, r)$.

To begin, set the coefficient of $d \bar{r}^{2}$ equal to zero in (3.14) to obtain 


$$
1+b\left\{b\left(-1+H^{2} \bar{r}^{2}\right)+2 H \bar{r}\right\}=0 .
$$

This simplifies to

$$
(H \bar{r} b+1)^{2}=b^{2},
$$

so that

$$
b=\frac{-1}{H \bar{r}_{-}^{+} 1} .
$$

In order to ensure that $b$, given in (3.16), is nonsingular and of a single sign, we choose the plus sign if $\dot{R}>0$, (the case of a White Hole explosion), and the minus sign if $\dot{R}<0$, (the case of collapse to a Black Hole). Matching the cross terms in (3.1) and (3.14) yeilds

$$
\frac{2}{\phi}\left\{-b\left(-1+H^{2} \bar{r}^{2}\right)-H \bar{r}\right\}=-2 \text {. }
$$

Using (3.15), we obtain

$$
\phi=H \bar{r}+\frac{1}{b}={ }_{+}^{-} 1
$$

at the shock surface. Here, consistent with (3.16), we take +1 if $H=\frac{\dot{R}}{R}>0$, and -1 if $H=\frac{\dot{R}}{R}<0$. Matching the $d \hat{t}^{2}$ terms in (3.1) and (3.14) gives

$$
-A=\frac{1}{\phi^{2}}\left(H^{2} \bar{r}^{2}-1\right)
$$

Since $k=0$ and $p=0$, the FRW equations (2.7), (2.8) imply

$$
H^{2}=\frac{\kappa}{3} \rho
$$

Using (3.20) and (3.2) together with $\phi^{2}=1$ in (3.19) yields the equation for the shock surface,

$$
M=\frac{\kappa}{6} \rho(t) \bar{r}^{3} .
$$

Solving (3.21) for $\bar{r}$ as a function of $t$ gives the shock surface $\bar{r}=\bar{r}(t)$ in the FRW coordinate. It follows from the FRW equations $(2.7),(2.8)$ in the case $p=0$, that $R(t)^{3} \rho(t)$ is constant on FRW solutions, [24]. Using this in (3.21) implies that $r=$ $r_{0}=$ const. describes the interface in the OS solution. For example, if we choose $R\left(t_{0}\right)=1$ at present time $t=t_{0}$, then 


$$
r_{0}=\left\{\frac{6 M}{\kappa \rho\left(t_{0}\right)}\right\}^{1 / 3}
$$

is constant on the interface, and thus $\bar{r}(t)=R(t) r_{0}$ describes the motion of the interface in the OS solution. The fact that $r=$ const. along the interface implies that the interface moves with the galaxies of the $p=0$ FRW metric, and hence neither mass nor momentum crosses the interface. From this we can also see that the shock surface is non-characteristic for the PDE (3.11). Indeed, the characteristic curves for (3.11) satisfy

$$
\frac{d \bar{r}}{d t}=-\frac{1}{b}=\left(\dot{R} r_{0}\right)_{-}^{+} 1
$$

on the shock surface, where the ${ }_{-}^{+}$agrees with the sign of $\dot{R}$. Thus we see that $\frac{d \bar{r}}{d t}=$ $\left(\dot{R} r_{0}\right)_{-}^{+} 1$ along the characteristic, and $\frac{d \bar{r}}{d t}=\dot{R} r_{0}$ along the shock surface, so the two can never be equal. We conclude that the shock surface is never characteristic for equation (3.11), and hence $\phi$, as well as the coordinate $\hat{t}$, can be defined in a neighborhood of the interface.

Note that the shock surface equation (3.21) tells us that $\bar{r}$ goes from zero to infinity as the FRW density goes from infinity to zero. For example in the case $\dot{R}>0$, this tells us that the FRW universe starts at the White Hole singularity of the EF metric at the instant $t=0$ of the Big Bang. From this time onward, the shock surface $\bar{r}(t)=\left(\frac{6 M}{\kappa \rho(t)}\right)^{1 / 3}$ continues out until the the Hubble distance $H^{-1}=\left(\frac{3}{\kappa \rho(t)}\right)^{1 / 2}$, (c.f. (3.20)), catches up to the shock surface on the FRW side of the shock, which happens at the FRW time $t_{s}$ when $\bar{r}\left(t_{s}\right)=2 M$, i.e., when the shock surface lies on the event horizon of the outer EF metric. As the FRW time increases from $t_{s}$ to $\infty$, the interface continues on out to infinity, staying inside the Hubble length on the FRW side of the shock, and outside the Black Hole on the EF side.

Note also that the coordinate $\bar{r}$ is a spacelike coordinate that measures arc-length distance in the FRW metric at fixed time $t$ in the FRW coordinate system $(t, \bar{r})$ on the FRW side of the shock, but on the EF side of the shock, the coordinate $\bar{r}$ is lightlike in $E F$ coordinates $(\hat{t}, \bar{r})$, and changes from timelike to spacelike in standard Schwarzschild coordinates $(\bar{r}, \bar{t})$ as the shock surface passes through the event horizon of the EF metric - even so, the coordinate identification $(t, r) \rightarrow(\hat{t}, \bar{r})$ is regular in a neighborhood of the shock surface for all shock positions $0<\bar{r}<\infty$. This is no contradiction, because the coordinate vector field $\frac{\partial}{\partial \bar{r}}$ remains undetermined until a choice of complementary coordinate is specified.

In conclusion, we have that the interface between the FRW and Schwarzschild metrics in the $k=0$ OS solution is a contact discontinuity that traverses a geodesic of the Schwarzschild metric. The total mass of the FRW metric behind the shock wave interface is finite and constant in time, and emerges from the White Hole singularity of the ambient Schwarzschild metric at $\bar{r}=0$, the instant of the Big Bang. After a finite proper time, the solution continues out through the event horizon at Schwarzschild time $t=-\infty$, and continues to expand forever into the asymptotically flat Schwarzshild spacetime outside the Black Hole, where $\frac{2 M}{\bar{r}}<1$. In this regime the solution agrees with the $k=0$ OS solution given in $[17]^{8}$.

\footnotetext{
${ }^{8}$ It is well known that the spacetime coordinatized by either the EF or Schwarzschild coordinates
} 
4. The TOV Solution Inside the Black Hole. The standard TolmanOppenheimer-Volkoff (TOV) metric models a static fluid sphere in general relativity. It was proved in [15] that the standard TOV metric for a static fluid sphere, by itself, does not admit Black Holes, and can only exist when $\frac{2 M}{\bar{r}}<1$. In this section we derive the equations for the analogue of the TOV solution inside the Black Hole.

The TOV solutions inside the Black Hole are used in Sections 5 and 6 to extend the OS solution of Section 3 to the case of non-zero pressure, inside the Black Hole. When $p \neq 0$, energy and momentum cross the interface, and so the contact discontinuity of the OS solution must be replaced by a shock wave discontinuity. Thus in order to extend our shock matching techniques beyond the Hubble length when $p \neq 0$, we must replace the outer Schwarzschild metric of the OS solution with a metric that contains matter, and satisfies $2 M / \bar{r}>1$. The TOV metric inside the Black Hole is the simplest metric that satisfies these conditions. We refer to it as TOV because the components depend only on the radial coordinate $\bar{r}$, as in the standard TOV metric, but now $\bar{r}$ is timelike. (As in the Schwarzschild metric, the roles of space and time are interchanged inside the Black Hole.) In shock matching with FRW metrics, this new TOV metric inside the Black Hole will play the role of a transitional solution that mediates the mass flux across the shock interface during the time after the Big Bang when the densities are large, and up until the time when the solution has settled down to a zero pressure OS expansion.

The usual ansatz for a TOV metric takes the form

$$
d s^{2}=-B d \bar{t}^{2}+A^{-1} d \bar{r}^{2}+\bar{r}^{2} d \Omega^{2}
$$

where $A(\bar{r})$ and $B(\bar{r})$ depend only on the coordinate $\bar{r}$. Here

$$
A(\bar{r})=1-\frac{2 M(\bar{r})}{\bar{r}},
$$

and when $\bar{r}>2 M, M(\bar{r})$ has the interpretation as the total mass inside the ball of radius $\bar{r}$. Thus our assumption that the TOV metric lies inside the Black Hole or inside the Schwarzschild radius is equivalent to the assumption that

$$
A<0 \text {. }
$$

We now obtain the Einstein equations for a perfect fluid under the assumption that the fluid is co-moving with respect to a metric of form (4.1), assuming that $A$ and $B$ depend only on $\bar{r}$, but now assuming that $A<0$. The stress tensor for a perfect fluid takes the form,

is not a geodesically complete spacetime. That is, from the point of view of the (complete) Kruskal development of the Schwarzschild spacetime, the EF metric covers only two of the four quadrants determined by the event horizon in the Kruskal diagram; namely, the standard quadrant outside the Black Hole together with the quadrant which contains the Black Hole singularity-or else under time reversal, the standard quadrant outside the Black Hole together with the region containing the White Hole singularity. On the other hand, the OS solution is a geodesically complete spacetime because, from the Kruskal point of view, it consists of the Schwarzschild solution on one side, and the FRW solution on the other side, of the geodesic defined by the trajectory of the interface of discontinuity. Thus, by removing a neighborhood of either the White Hole or the Black Hole event horizon, the OS matching also eliminates the incomplete geodesics that emerge from $t=-\infty$, respectively $t=+\infty$, depending on the time orientation of the solution, [9]. 


$$
T_{i j}=(\bar{\rho}+\bar{p}) \bar{w}_{i} \bar{w}_{j}+\bar{p} \bar{g}_{i j},
$$

and since $\bar{r}$ is the timelike coordinate for the TOV metric in $(\bar{r}, \bar{t})$-coordinates when $A<0$, the assumption that the fluid is co-moving with the TOV metric inside the Black Hole implies that, c.f. [24],

$$
\left(\bar{w}_{0}, \bar{w}_{1}, \bar{w}_{2}, \bar{w}_{3}\right)=\left(\bar{w}_{\bar{r}}, \bar{w}_{\bar{t}}, \bar{w}_{\theta}, \bar{w}_{\phi}\right)=\left(\frac{1}{\sqrt{-A}}, 0,0,0\right) .
$$

In this case we obtain

$$
T_{00} \equiv T_{\bar{r} \bar{r}}=(\bar{\rho}+\bar{p})\left(\frac{1}{-A}\right)+\bar{p}\left(\frac{1}{A}\right)=-\frac{\bar{\rho}}{A},
$$

and

$$
T_{11} \equiv T_{\bar{t} \bar{t}}=\bar{p} \bar{g}_{11}=-\bar{p} B
$$

Using MAPLE we find,

$$
\begin{gathered}
G_{00} \equiv G_{\bar{r} \bar{r}}=\frac{\bar{r} A B^{\prime}-B+A B}{\bar{r}^{2} A B}, \\
G_{11} \equiv G_{\overline{t \bar{t}}}=-\frac{B}{\bar{r}^{2}}\left\{\bar{r} A^{\prime}-1+A\right\} .
\end{gathered}
$$

From (4.5)-(4.8), the Einstein equations $G_{00}=\kappa T_{00}$ and $G_{11}=\kappa T_{11}$ reduce to

$$
\frac{B^{\prime}}{B}=\frac{1}{\bar{r}}\left(\frac{1-A}{A}\right)-\kappa \frac{\bar{\rho} \bar{r}}{A},
$$

and

$$
A^{\prime}=\frac{1-A}{\bar{r}}+\kappa \bar{p} \bar{r}
$$

respectively. ${ }^{9}$ From [24], equation (5.45), we also have

$$
-\bar{p}^{\prime}=(\bar{p}+\bar{\rho}) \frac{d}{d \bar{r}} \ln \left\{\frac{1}{\sqrt{-A}}\right\},
$$

which simplifies to

\footnotetext{
${ }^{9}$ Beware that MAPLE's convention is that $-G_{M A P L E}=G$, so the Einstein equations are $-G_{M A P L E}=\kappa T$.
} 


$$
\bar{p}^{\prime}=\frac{\bar{p}+\bar{\rho}}{2} \frac{A^{\prime}}{A}=\frac{\bar{p}+\bar{\rho}}{2 A}\left\{\frac{1-A}{\bar{r}}+\kappa \bar{p} \bar{r}\right\} .
$$

Using (4.2), we obtain the system

$$
\begin{aligned}
\bar{p}^{\prime} & =\frac{\bar{p}+\bar{\rho}}{2} \frac{A^{\prime}}{A}=\frac{\bar{p}+\bar{\rho}}{2 A}\left\{\frac{1-A}{\bar{r}}+\kappa \bar{p} \bar{r}\right\}, \\
A^{\prime} & =\frac{1-A}{\bar{r}}+\kappa \bar{p} \bar{r}, \\
\frac{B^{\prime}}{B} & =\frac{1}{\bar{r}}\left(\frac{1-A}{A}\right)-\kappa \frac{\bar{\rho} \bar{r}}{A} .
\end{aligned}
$$

Alternatively, using the unknown $N=1-A$ instead of $A$, (a variable convenient for our subsequent analysis), we obtain the equivalent system,

$$
\begin{aligned}
\bar{p}^{\prime} & =\frac{\bar{p}+\bar{\rho}}{2} \frac{N^{\prime}}{N-1}, \\
N^{\prime} & =-\left\{\frac{N}{\bar{r}}+\kappa \bar{p} \bar{r}\right\}, \\
\frac{B^{\prime}}{B} & =-\frac{1}{N-1}\left\{\frac{N}{\bar{r}}-\kappa \bar{\rho} \bar{r}\right\} .
\end{aligned}
$$

Note that the essential reason why the equations for the TOV metric inside the Black Hole take a different form than the standard TOV equations "outside the Black Hole", is that the assumption of co-moving puts the nonzero component of $\mathbf{w}$ on the timelike coordinate $\bar{r}$ when $A<0$, and on the timelike coordinate $\bar{t}$ when $A>0$. We conclude that system (4.16)-(4.18) defines a new class of gravitational metrics which describe spacetimes that evolve inside the Black Hole.

The TOV equations inside the Black Hole describe a time dependent metric in which the metric components, together with the fluid variables, are constant at each fixed value of the TOV timelike coordinate $\bar{r}$. Like the FRW metric, the TOV metric inside the Black Hole describes a fluid with pressure that emerges from a White Hole singularity at $\bar{r}=0$, the instant of the Big Bang, with one important difference: unlike the FRW metric, for the TOV metric inside the Black Hole, the total mass is constant on each spacelike slice $\bar{r}=$ constant. In the next section we match the TOV metric inside the Black Hole to $k=0$ FRW metrics across shock interfaces in order to make the expanding FRW universe finite, in the sense that the total mass on each spacelike hypersurface is finite.

5. Shock Matching Inside the Black Hole. In this section we derive the equations that describe the matching of a general $k=0 \mathrm{FRW}$ metric to a TOV metric inside the Black Hole, at a shock wave interface across which energy and momentum are conserved. The equations, given in system (5.35)-(5.36) below, describe the simultaneous time evolution of the shock position together with the TOV metric inside the Black Hole such that the resulting metric matches a given $k=0 \mathrm{FRW}$ metric Lipschitz continuously across the shock, and such that conservation of energy and momentum hold at the shock. The conservation constraint, given in (5.25) below, 
determines the TOV pressure, and this is used to close the equations, c.f [14]. Equations (5.35)-(5.36) then determine all other unknowns in the TOV metric beyond the shock, and guarantee conservation, once the FRW metric is assigned. In particular, the equations guarantee that there are no delta function sources produced by the (second order) Einstein equations due to the lower order smoothness (Lipschitz continuity) of the metrics at the shock, c.f. (i)-(iv) below. The success of the method relies on the fact that once Lipschitz continuity is imposed, the single conservation condition (5.25) alone guarantees the two conservation constraints of the Rankine-Hugoniot jump conditions at the shock, c.f. [13], and Theorem 3 below.

Solutions of equations (5.35)-(5.36) are formally time-reversible without the imposition of an entropy condition. In the next section we formulate an entropy condition that agrees with the entropy condition of gas dynamics, and we use this to construct exact solutions of (5.35)-(5.36) that describe a class of time-irreversible entropy satisfying shocks in which the TOV density and pressure satisfy the physical bounds $0<\bar{p}<\bar{\rho}$.

To model the expanding universe with a spherical shock wave at FRW position $\bar{r}=\bar{r}(t)$, we assume that the FRW metric lies in the region $\bar{r}<\bar{r}(t)$, and the TOV metric lies beyond the shock wave at $\bar{r}>\bar{r}(t)$. The corresponding matching for a standard TOV metric outside the Black Hole, (and inside the Hubble length), was accomplished in $[14,15,21]$. In this section we do not use an EF type regularization of the TOV metric, (c.f. (2.9)), but rather we employ standard Schwarzschild coordinates, avoiding the singularity at $A=0$ by working either inside or outside the Black Hole separately.

Our proceedure for shock matching is as follows: We first identify the shock surface across which a $k=0$ FRW metric matches a TOV metric inside the Black Hole, such that the matching is Lipschitz continuous across the interface, and such that we have a smooth matching of the spheres of symmetry, (c.f., [13], Lemma 9, equation (5.3)). The Lipschitz matching of the metrics is guaranteed by a non-characteristic condition that always holds outside the Black Hole, when $A>0$, c.f. (5.23) below. Given this matching, we then determine a conservation constraint that guarantees that the Rankine-Hugoniot jump conditions

$$
\left[T^{\mu \nu}\right] n_{\mu}=0,
$$

hold across the shock. (Here, as usual, [·] denotes the jump in a quantity across the shock interface, $n_{\mu}$ are the covariant components of the normal vector $\mathbf{n}$ to the shock surface, and assuming spherical symmetry, we need only require (5.1) for $\nu=0,1$.) We can then apply [13], Lemma 9, which states that for metrics matched Lipschitz continuously across the shock, with a smooth matching of the spheres of symmetry, the Rankine-Hugoniot jump conditions (5.1) imply that the following equivalencies are also valid at the shock:

(i) The extrinsic curvature is continuous across the shock,

(ii) The Riemann and Einstein curvature tensors, viewed as second order operators on the metric components, produce no delta function sources on the shock,

(iii) In a neighborhood of each point on the shock surface there exist coordinate transformations such that in the new coordinates, all second derivatives of the metric components are bounded, and

(iv) At each point on the shock surface there exist coordinate frames that are locally Lorentzian. 
In the papers $[13,14,17,21]$, the main idea for carrying out this procedure in the case $A>0$, was to show that the single condition

$$
\left[T^{\mu \nu}\right] n_{\mu} n_{\nu}=0,
$$

alone implies the jump conditions (5.1), and hence (i)-(iv). However, we must modify this idea in the case $A<0$ because it turns out that the condition (5.2) has a nonphysical solution which everywhere violates the non-characteristic condition used to guarantee the Lipschitz matching of the metrics at the shock. For this reason, to obtain the conservation condition when the shock wave lies inside the Black Hole, we verify the Rankine-Hugoniot conditions directly. That is, we derive a conservation constraint equivalent to the condition $\operatorname{det}[T]=0$, (instead of (5.2)), and then show that the normal $\mathbf{n}$ to the shock surface is in the kernel of $[T]$ when $\operatorname{det}[T]=0$. This then implies that when the conservation constraint holds at the shock, the jump conditions $\left[T^{\mu \nu}\right] n_{\mu}=0$ hold, and then Lemma 9 of [13] implies that (i)-(iv) also hold at the shock.

To start, we match the FRW to the TOV metric when $k=0$ and $A<0$. The $k=0 \mathrm{FRW}$ metric in the usual $(t, r)$ coordinates is given by,

$$
d s^{2}=-d t^{2}+R(t)^{2}\left\{d r^{2}+r^{2} d \Omega^{2}\right\} .
$$

In (2.18)-(2.20) we showed that the mapping $(t, r) \rightarrow(\bar{r}, \bar{t})$ that takes metric (5.3) to standard Schwarzschild coordinates in which the metric takes the form,

$$
d s^{2}=-B_{F R W} d \bar{t}^{2}+A_{F R W}^{-1} d \bar{r}^{2}+\bar{r}^{2} d \Omega^{2},
$$

is given by

$$
\bar{r}=R(t) r
$$

and

$$
d \bar{t}=(\psi C) d t-(\psi E) d \bar{r},
$$

where

$$
\begin{aligned}
& C=1-H^{2} \bar{r}^{2}, \\
& E=-\bar{r} H .
\end{aligned}
$$

(Recall that $\frac{\partial}{\partial \bar{r}}$ is timelike in the coordinate system $(\bar{r}, \bar{t})$ and spacelike in the coordinate system $(t, \bar{r})$ when $A_{F R W}<0, H^{-1}>1$.) Here $\psi$ can be taken to be any solution of

$$
\frac{\partial}{\partial \bar{r}}(\psi C)+\frac{\partial}{\partial t}(\psi E)=0,
$$

determined from initial data on any non-characteristic surface, (c.f. the paragraph following (2.21)). Under this coordinate transformation, we obtain 


$$
\begin{aligned}
A_{F R W}^{-1} & =\frac{R^{2}}{R^{2}-\bar{r}^{2} \dot{R}^{2}}=\frac{1}{1-(\bar{r} H)^{2}} \\
B_{F R W} & =\frac{1}{\psi^{2}} \frac{R^{2}}{R^{2}-\bar{r}^{2} \dot{R}^{2}}=\frac{1}{\psi^{2}} \frac{1}{1-(\bar{r} H)^{2}}
\end{aligned}
$$

c.f. (2.18)-(2.20). We use the subscript $F R W$ to distinguish the FRW metric coefficients $A_{F R W}=1-\frac{2 M_{F R W}}{\bar{r}}$ and $B_{F R W}$ from the TOV metric coefficients $A=1-\frac{2 M}{\bar{r}}$ and $B$, that appear in the TOV line element

$$
d s^{2}=-B d \bar{t}^{2}+A^{-1} d \bar{r}^{2}+\bar{r}^{2} d \Omega^{2},
$$

in standard Schwarzschild coordinates. By shock matching, using (2.7) and (5.10), we find that

$$
M_{F R W}(t, \bar{r})=\frac{1}{2} \bar{r}^{3} H^{2}=\frac{\kappa}{6} \rho(t) \bar{r}^{3} .
$$

Assume now that the $(\bar{r}, \bar{t})$ coordinates that describe the TOV and FRW metrics in standard Schwarzschild coordinates, actually represent a single coordinate system for the pair of metrics matched across a shock surface where the metrics agree; that is, where $A=A_{F R W}$ and $B=B_{F R W}$. Setting $A_{F R W}=A$ and using (5.13) gives

$$
\frac{2 M_{F R W}}{\bar{r}}=(H \bar{r})^{2}=\frac{\kappa}{3} \rho \bar{r}^{2}=\frac{2 M}{\bar{r}},
$$

from which we deduce the following conservation of mass condition that must hold at the shock surface, and which is independent of $\psi$ :

$$
\frac{\kappa}{6} \rho(t) \bar{r}^{3}=M(\bar{r}) .
$$

Equation (5.15) implicitly defines the shock surface $\bar{r}=\bar{r}(t)$, which then determines the position $r=r(t)=\frac{\bar{r}(t)}{R(t)}$ of the shock in the original FRW coordinates $(t, r)$. By (5.14) we also see that

$$
N \equiv 1-A=\frac{2 M}{\bar{r}}=(\bar{r} H)^{2},
$$

and so

$$
\bar{r}=\sqrt{N} H^{-1}
$$

holds on the shock surface. Using (5.17), we can interpret $N$ from the TOV metric as follows: if $(t, \bar{r})$ is a point on the shock surface, then $\sqrt{N(t, \bar{r})}$ is equal to the number of Hubble lengths from the center of the FRW spacetime to the shock surface as measured at fixed time $t$ on the FRW side of the shock. Since $N>1$ if and only if $A<0$, it follows that the shock wave lies beyond one Hubble length from the FRW center when $A<0$. Note that $N$ is a convenient variable because it appears in system (4.16)-(4.18). 
Setting $B_{F R W}=B$ at the shock surface and using (5.11), we obtain the initial condition for the integrating factor $\psi$ of the FRW metric on the shock surface (5.15); namely,

$$
\psi={ }_{-}^{+}\left\{\left(1-H^{2} \bar{r}^{2}\right) B(\bar{r})\right\}^{-1 / 2}=_{-}^{+}\{A B\}^{-1 / 2},
$$

where we have used that $1-H^{2} \bar{r}^{2}=A<0$ at the shock. Note that $A B>0$, and the choice of sign in (5.18) determines the time orientation for $\bar{t}$, c.f. (5.6).

We now derive a condition that guarantees that the surface $\bar{r}=\bar{r}(t)$ is noncharacteristic when $A<0 .{ }^{10}$ To this end, differentiate (5.15) with respect to FRW time $t$ to obtain,

$$
M^{\prime} \dot{\bar{r}}=\frac{\kappa}{6} \dot{\rho} \bar{r}^{3}+\frac{\kappa}{2} \rho \bar{r}^{2} \dot{\bar{r}}
$$

Using (2.8) and (4.17) in (5.19) we obtain the following formula for the speed $\dot{\bar{r}}$, the speed at which the shock wave is receding from an observer fixed at the FRW origin:

$$
\dot{\bar{r}}=\frac{\rho+p}{\rho+\bar{p}} H \bar{r} .
$$

For future reference we record that this directly implies

$$
\dot{r}=\frac{H \bar{r}}{R}\left(\frac{p-\bar{p}}{\rho+\bar{p}}\right) .
$$

On the other hand, in light of (2.21), the characteristics for (5.9) satisfy

$$
\dot{\bar{r}}=-\{\bar{r} H\}^{-1}+\bar{r} H
$$

Thus the shock surface is characteristic if and only if the two speeds in (5.20) and (5.22) are equal, which holds if and only if

$$
1-\left\{\frac{\rho+p}{\rho+\bar{p}}\right\}=\frac{1}{(H \bar{r})^{2}} .
$$

Using (5.14) in (5.23) we conclude that the shock surface is non-characteristic for the PDE (5.9) if and only if

$$
A \neq \frac{\rho+p}{p-\bar{p}}
$$

\footnotetext{
${ }^{10}$ This is no moot point. Indeed, in previous work, (c.f. [13, 14]), the authors used (5.2) to obtain the conservation constraint across the shock surface when $A>0$, but we must be careful here because a calculation shows that one of the solutions of (5.2) is everywhere characteristic for (5.9) when $A<0$. It follows that (5.1) does not hold across the characteristic surface, and so there is a solution of (5.2) that does not represent an actual weak solution of $G=\kappa T$ when $A<0$. It is for this reason that we go directly to (5.1) to construct the conservation constraint in the case $A<0$ below.
} 
The inequality (5.24) is not in general ruled out on a timelike shock surface when $A<0$. However, (5.24) immediately implies the following lemma which we record for use in Section 6:

Lemma 1. If $\rho, p, \bar{\rho}, \bar{p}$ are all positive on the shock surface $\bar{r}=\bar{r}(t)$ defined implicitly by (5.15), then the surface is non-characteristic for the PDE (5.9) so long as $p>\bar{p}$ and $A<0$.

Assuming that (5.24) holds, the above procedure defines a mapping $(t, r) \rightarrow(\bar{r}, \bar{t})$, defined in a neighborhood of any point on the shock surface $(\bar{r}(t), t)$, such that, under this coordinate identification, the FRW metric matches the TOV metric Lipshitz continuously across the shock surface. Note also that as in the OS solution, the coordinate $\bar{r}$ is timelike in the Schwarzschild coordinates $(\bar{r}, \bar{t})$ when $A<0$, but spacelike in the FRW coordinates $(t, \bar{r})$, where the coordinate $\bar{r}$ measures arclength distance in each spatial slice $t=$ const.

It remains to analyze the Rankine-Hugoniot jump conditions (5.1). The following theorem gives a formulation of the conservation constraint that is amenable to analysis.

TheOREm 3. Assume that the coordinate mapping $(t, r) \rightarrow(\bar{r}, \bar{t})$ defines a Lipshitz continuous matching of an FRW metric to a TOV metric inside the Black Hole, in a neighborhood of a point $P$ across a non-characteristic shock surface $\bar{r}=\bar{r}(t)$ defined by (5.15), so that that $N>1$ at $P$. Assume further that $p, \rho>0$ at $P$. Then the Rankine-Hugoniot jump relations (5.1) together with the equivalent conditions (i)(iv) listed after (5.1)) all hold at $P$, if and only if the following single conservation constraint holds at the point $P$ :

$$
\bar{p}=\frac{p-\left(\frac{\bar{\rho}+p}{\rho-\bar{\rho}}\right) \frac{1}{N} \rho}{1+\left(\frac{\bar{\rho}+p}{\rho-\bar{\rho}}\right) \frac{1}{N}} .
$$

Note that (5.25) immediately implies that if $\rho>\bar{\rho}$, then also $p>\bar{p}$, consistent with an outgoing explosion of an inner FRW metric into an outer TOV metric. (The construction of an explicit example of such a shock wave is the topic of the next section.) Solving for $\bar{\rho}$, it follows that condition (5.25) is equivalent to the condition

$$
\bar{\rho}=\frac{-(\rho+\bar{p}) p+(p-\bar{p}) N \rho}{(\rho+\bar{p})+(p-\bar{p}) N} .
$$

Note also that from (5.26) we see that if $p=\bar{p}=0$, then also $\bar{\rho}=0$, the conditions of the OS solution.

Before giving the proof of Theorem 3, we first use the conservation constraint (5.26) to derive the equations that describe the time evolution of the shock surface and TOV metric inside the Black Hole that matches a given $k=0$ FRW metric at a shock wave positioned beyond one Hubble length $N>1$. To start, assume that $\rho(t), p(t)$ and $R(t)$ are known functions of the FRW time $t$ that determine a unique equation of state $p(\rho)$, and let $\sigma(t)=p(t) / \rho(t)$. The matching condition (5.15) written in the form

$$
\rho=\frac{3 N}{\kappa \bar{r}^{2}},
$$


gives $\rho$ as a function of $N$ and $\bar{r}$ at the shock, and substituting this into the conservation constraint (5.26) and using $p=p(\rho)$ gives $\bar{\rho}$ as a function of $\bar{p}, N$ and $\bar{r}$. The first two equations (4.16), (4.17) of the TOV system (4.16)-(4.18) then close upon substituting the resulting expression $\bar{\rho}=\bar{\rho}(\bar{p}, N, \bar{r})$ for $\bar{\rho}$ in the first equation (4.16). The resulting system,

$$
\begin{aligned}
\bar{p}^{\prime} & =\frac{\bar{p}+\bar{\rho}(\bar{p}, N, \bar{r})}{2} \frac{N^{\prime}}{N-1}, \\
N^{\prime} & =-\left\{\frac{N}{\bar{r}}+\kappa \bar{p} \bar{r}\right\},
\end{aligned}
$$

then forms a non-autonomous system of two equations in the unknowns $(\bar{p}, N)$ as a function of the independent variable $\bar{r}$, the shock position. (Again, "prime" denotes $\frac{d}{d \bar{r}}$.) Solving $(5.28),(5.29)$ gives $\bar{p}(\bar{r})$ and $N(\bar{r})$ subject to the initial conditions

$$
\bar{p}=\bar{p}_{0}, \quad N=N_{0}, \text { at } \bar{r}=\bar{r}_{0} .
$$

Assuming the FRW solution $\rho(t), p(t)$, and $R(t)$ is given, we can obtain $\bar{r}$ as a function of $t$ from (5.27), whereby we conclude that $\bar{p}(\bar{r})$ and $N(\bar{r})$ determine the entire shock wave solution. For example, to set the position of the shock at present time in the FRW metric to be at $\bar{r}=\bar{r}_{0}$, choose $\rho_{0}=$ present density, define

$$
N_{0}=\frac{\kappa}{3} \rho_{0} \bar{r}_{0}^{2}
$$

(c.f. use (5.27)). This leaves $\bar{p}_{0}$ as a free parameter. Once we know the solution $(\bar{p}(\bar{r}), N(\bar{r}))$, we can use (5.27) to obtain the FRW density $\rho$ as a function of $\bar{r}$, and inverting the relation

$$
\rho(t)=\frac{3 N(\bar{r})}{\kappa \bar{r}^{2}}
$$

gives the shock position $\bar{r}=\bar{r}(t)$, the distance from the shock wave to the FRW center at FRW time $t$. The FRW shock position is then

$$
r(t)=\frac{\bar{r}(t)}{R(t)}
$$

The only other unknown in the problem is the metric coefficient $B(\bar{r})$ from the TOV metric, which we get by integrating the equation $(4.18)^{11}$,

$$
B(\bar{r})=B_{0} \exp \left\{-\int_{\bar{r}_{0}}^{\bar{r}} \frac{1}{N(\xi)-1}\left(\frac{N(\xi)}{\xi}-\kappa \bar{\rho}(\xi) \xi\right) d \xi\right\}
$$

\footnotetext{
${ }^{11}$ Note that (5.33) completes the definition of the TOV metric, and so the integrating factor $\psi$ for the shock matching is determined by (5.18), and from this the non-characteristic condition (5.24) implies the Lipschitz matching of the metrics.
} 
Note that for an outgoing shock wave in an expanding FRW metric, it follows from (5.29) that $N$ decreases and $\bar{r}$ increases in forward FRW time, and so $N \rightarrow \infty, \bar{r} \rightarrow 0$ would correspond to the Big Bang from the FRW point of view.

We conclude that the problem of constructing FRW-TOV shock waves with $N>1$ reduces to the analysis of the $2 \times 2$ system (5.28), (5.29). The next theorem shows that when we make the change of variable $\bar{p} \rightarrow u=\frac{\bar{p}}{\rho}$ and take $N$ to be the independent variable, the resulting equivalent system has the nice property that the equations are coupled to the FRW metric only through the variable $\sigma=\frac{p}{\rho}$.

THEOREM 4. Under the change of variables

$$
u=\frac{\bar{p}}{\rho}, v=\frac{\bar{\rho}}{\rho}, \sigma=\frac{p}{\rho},
$$

system (5.28), (5.29) with conservation constraint (5.26) transforms to the equivalent system

$$
\begin{gathered}
\frac{d u}{d N}=-\left\{\frac{(1+u)}{2(1+3 u) N}\right\}\left\{\frac{(3 u-1)(\sigma-u) N+6 u(1+u)}{(\sigma-u) N+(1+u)}\right\}, \\
\frac{d \bar{r}}{d N}=-\frac{1}{1+3 u} \frac{\bar{r}}{N},
\end{gathered}
$$

with conservation constraint

$$
v=\frac{-\sigma(1+u)+(\sigma-u) N}{(1+u)+(\sigma-u) N} .
$$

For such solutions, the speed of the shock interface relative to the fluid comoving on the FRW side of the shock, is given by

$$
s=R \dot{r}=\sqrt{N}\left(\frac{\sigma-u}{1+u}\right) .
$$

Note that the dependence of (5.35)-(5.37) on the FRW metric is only through the variable $\sigma$, and hence (5.35) is coupled to (5.36) only through the function $\sigma$. In particular, if we assume that $\sigma$ is constant in the FRW metric, (a reasonable model problem, c.f. [14]), then equation (5.35) uncouples from equation (5.36) to form a nonautonomous scalar equation in $u$ and $N$ which is amenable to phase plane analysis. This is the starting point for the exact solutions constructed in the next section.

Note too that since (5.36) implies that $\frac{d N}{d \bar{r}}<0$, and $\sqrt{N}$ gives the number of Hubble lengths to the shock wave, c.f. (5.17), equation (5.36) implies that the number of Hubble lengths from the FRW center to the shock wave decreases in time, as claimed in the introduction.

Since system system (5.35), (5.36) is equivalent to system (5.28), (5.29), the former system also determines all quantities in the shock wave solution, but now as a function of the variable $N$. For example, a solution of system (5.35), (5.36) is determined by the initial conditions $u=u_{0}$ and $\bar{r}=\bar{r}_{0}$ at $N=N_{0}$, which entails assigning three constants. Alternatively, if we ask that the shock wave be positioned $\sqrt{N_{0}}$ Hubble lengths from the FRW center at the present FRW value of the density $\rho=\rho_{0}$, then by $(5.27)$, the initial shock position is determined by 


$$
\bar{r}_{0}=\sqrt{\frac{3 N_{0}}{\kappa \rho_{0}}},
$$

and there is then a one parameter family of such solutions $(u(N), \bar{r}(N))$ determined by the remaining free parameter

$$
u_{0}=\frac{\bar{p}_{0}}{\rho_{0}} .
$$

Once we know the solution $(u(N), \bar{r}(N))$, we can use

$$
\rho=\frac{3 N}{\kappa[\bar{r}(N)]^{2}},
$$

to obtain $\rho(N)$, the FRW density $\rho$ as a function of $N$ at the shock. Since the FRW metric is known, knowing $\rho(N)$ determines $p(N)$ and $\sigma(N)=\frac{p(N)}{\rho(N)}$, and using these in (5.37) we obtain $v(N)$ and $\bar{\rho}(N)=v(N) \rho(N)$. Finally, the TOV metric coefficient $B$ is determined as a function of $N$ by integrating the equation (4.18) with respect to $N$, yielding the formula

$$
B(\bar{r})=B_{0} \exp \left\{-\int_{N_{0}}^{N} \frac{1}{\xi-1}\left(\frac{\xi}{\bar{r}(\xi)}-\kappa \bar{\rho}(\xi) \bar{r}(\xi)\right) d \xi\right\} .
$$

To connect $N$ with the FRW time $t$, invert the relation (5.41), obtaining $N(t)$. Since $R(t)$ is assumed known, we can use

$$
r(t)=\frac{\bar{r}(N(t))}{R(t)}
$$

to get the FRW shock position $r$ as a function of $t$. Relations (5.39)-(5.43) formally determine an exact shock wave solution of the Einstein equations for any given $(k=0)$ FRW metric, and any given solution of system (5.35), (5.36).

Proof of Theorem 4. Equation (5.37) follows directly from (5.26) upon dividing through by $\rho$ and making the substitutions in (5.34). Equation (5.36) follows directly from (4.17) upon noting that $\kappa \bar{p} \bar{r}=\frac{3 u N}{\bar{r}}$, a consequence of (5.32). From (5.36) we have

$$
\frac{1}{\bar{r}}=-\frac{1}{(1+3 u)} \frac{N^{\prime}}{N}
$$

To verify (5.35), start with

$$
u^{\prime}=\left(\frac{\bar{p}}{\rho}\right)^{\prime}=\frac{\bar{p}^{\prime}}{\rho}-\frac{\bar{p}}{\rho^{2}} \rho^{\prime}
$$


Then by (5.28) and (5.37),

$$
\frac{\bar{p}^{\prime}}{\rho}=\frac{u+v}{2} \frac{N^{\prime}}{N-1}=\frac{1}{2}\left\{\frac{(1+u)(\sigma-u)}{(1+u)+(\sigma-u) N}\right\} N^{\prime}
$$

and using (5.27),

$$
\rho^{\prime}=\left\{\frac{3 N}{\kappa \rho \bar{r}^{2}}\right\}^{\prime}=\rho\left(\frac{N^{\prime}}{N}-\frac{2}{\bar{r}}\right)=\rho\left(\frac{3(1+u)}{1+3 u}\right) \frac{N^{\prime}}{N},
$$

where we have used (5.44). Thus,

$$
\frac{\bar{p}}{\rho^{2}} \rho^{\prime}=\left(\frac{3 u(1+u)}{1+3 u}\right) \frac{N^{\prime}}{N}
$$

and so using (5.46) and (5.48) in (5.45) gives

$$
\begin{aligned}
u^{\prime} & =\frac{1}{2}\left\{\frac{(1+u)(\sigma-u)}{(1+u)+(\sigma-u) N}\right\} N^{\prime}-\left(\frac{3 u(1+u)}{1+3 u}\right) \frac{N^{\prime}}{N} \\
& =-\frac{(\sigma-u)\left(3 u^{2}+2 u-1\right) N+2 u(1+u)^{2}}{2(1+3 u)[(1+u)+(\sigma-u) N] N} N^{\prime}, \\
& =-\left\{\frac{(1+u) N^{\prime}}{2(1+3 u) N}\right\}\left\{\frac{(3 u-1)(\sigma-u) N+6 u(1+u)}{(\sigma-u) N+(1+u)}\right\},
\end{aligned}
$$

which upon dividing by $N^{\prime}$ verifies (5.35). Finally, since the fluid on the FRW side of the shock is assumed to be co-moving with the radial coordinate $r$ of the FRW coordinate system, it follows that $s=R \dot{r}$ gives the speed of the shock relative to the FRW fluid, (c.f., the discussion after Theorem 4 of [14]), and so (5.38) follows directly from (5.21).

Proof of Theorem 3. Assume that we are given an FRW metric and TOV metric inside the Black Hole that match Lipshitz continuously across a smooth, noncharacteristic shock surface $\bar{r}=\bar{r}(t)$ defined in a neighborhood of point $P$, such that the hypotheses of Theorem 3 hold. We show that (5.1) holds if and only if (5.25) holds. We use the following lemma:

LEMMA 2. On the shock surface, in $(\bar{r}, \bar{t})$ coordinates, we have,

$$
\begin{aligned}
{[T]^{\mu \nu} } & \equiv\left[T_{F R W}^{\mu \nu}-T_{T O V}^{\mu \nu}\right] \\
& =\left[\begin{array}{cr}
(\rho+p) N+(\bar{\rho}+p)(1-N) & \psi \sqrt{N}(\rho+p) \\
\psi \sqrt{N}(\rho+p) & \psi^{2}\{(\rho+\bar{p})+(p-\bar{p}) N\}
\end{array}\right],
\end{aligned}
$$

and

$$
\begin{aligned}
& \bar{n}_{0}=\psi\left(N \frac{p-\bar{p}}{\rho+\bar{p}}+1\right), \\
& \bar{n}_{1}=-\sqrt{N}\left(\frac{\rho+p}{\rho+\bar{p}}\right),
\end{aligned}
$$


where $\bar{n}_{\mu} d \bar{x}^{\mu}=\bar{n}_{0} d \bar{r}+\bar{n}_{1} d \bar{t}$ is the covariant normal to the shock surface.

We use Lemma 2 to verify Theorem 3, and postpone the proof of Lemma 2 until the end. To verify that (5.1) is equivalent to (5.25) at a point $P$ on the shock surface, it suffices to show that $\operatorname{det}\left([T]^{\mu \nu}\right)=0$ holds at $P$ if and only if (5.25) holds at $P$, and that when this holds, the shock normal $\mathbf{n}$ is in the kernel of $[T]$. Indeed, if (5.25) holds at $P$, and we know $\mathbf{n}$ is in the kernel of $[T]^{\mu \nu}$, then (5.1) and (5.2) hold at $P$, and so our general theory in [13] would imply that (i)-(iv) hold at $P$ as well. Conversely, if (i)-(iv) and (5.1) all hold at $P$, then the RH jump conditions (5.1) alone imply that $\operatorname{det}\left([T]^{\mu \nu}\right)=0$ holds at $P$, and hence we would have (5.25) at $P$ as well.

We first show that $\operatorname{det}\left([T]^{\mu \nu}\right)=0$ holds at $P$ if and only if (5.25) holds at $P$. But by (5.49),

$$
\begin{gathered}
\operatorname{det}\left([T]^{\mu \nu}\right)=\{(\rho+p) N+(\bar{\rho}+p)(1-N)\}_{I}\left\{\psi^{2}[(\rho+\bar{p})+(p-\bar{p}) N]\right\} \\
-\psi^{2} N(\rho+p)^{2}=0
\end{gathered}
$$

and solving for $\bar{p}$ gives

$$
\begin{aligned}
\bar{p} & =\left[-\frac{N(\rho+p)^{2}}{\{(\rho-\bar{\rho}) N+\bar{\rho}+p\}_{I}}+(\rho+p N)\right] \frac{1}{N-1} \\
& =\frac{\left\{(\rho+p N)\{\cdot\}_{I}-N(\rho+p)^{2}\right\}}{(N-1)\{\cdot\}_{I}} \\
& =\frac{(N-1)\{p(\rho-\bar{\rho}) N-\rho(\bar{\rho}+p)\}}{(N-1)\{(\bar{\rho}+p)+(\rho-\bar{\rho}) N\}}
\end{aligned}
$$

from which (5.25) follows at once. (Note that we have assumed without loss of generality that $\{\cdot\}_{I} \neq 0$, which is valid because, if $\{\cdot\}_{I}=0$, then (5.52) implies $\rho=p=0$, and $\rho=0$ violates $N>1$, e.g., $\dot{R} \neq 0$, c.f. (5.52), (5.16).) Thus it remains only to show that if $\operatorname{det}\left([T]^{\mu \nu}\right)=0$ holds at $P$, then the shock normal $\mathbf{n}$ at $P$ is in the kernel of $[T]$. To this end, note that by (5.50) and (5.51),

$$
\begin{aligned}
\bar{n}_{\mu}[T]^{\mu 1}= & \psi^{2}\left\{\left(N \frac{p-\bar{p}}{\rho+\bar{p}}+1\right) \sqrt{N}(\rho+p)\right. \\
& -\sqrt{N}\left(\frac{\rho+p}{\rho+\bar{p}}\right)((\rho+\bar{p})+(p-\bar{p}) N\}=0
\end{aligned}
$$

and so $\bar{n}_{\mu}[T]^{\mu 0}=0$ as well because we assume $\operatorname{det}[T]=0$, and $[T]$ is a $2 \times 2$ matrix 12. The proof of Theorem 3 is complete, once we give the

Proof of Lemma 2. To verify (5.49), we calculate $T_{T O V}^{\mu \nu}$ and $T_{F R W}^{\mu \nu}$, the stress tensors in barred coordinates $\overline{\mathbf{x}}=(\bar{r}, \bar{t})$, at the shock. (Recall that $\bar{x}^{0}=\bar{r}$ is the timelike coordinate, and $\bar{x}^{1}=\bar{t}$ is spacelike because $N>1$.) Using the formula

\footnotetext{
${ }^{12}$ Although (5.53) appears to be a miracle here, this is to be expected from [13, 22], where in the case $A>0$, the single condition $\left[T^{\mu \nu}\right] n_{\mu} n_{\nu}=0$ alone implied the equivalencies (i)-(iv), as well as the Rankine-Hugoniot conditions (5.1). One also expects this in light of the fact that the Einstein equations for a spherically symmetric metric in standard Schwarzschild coordinates, contains only one second order, (i.e. for Lipschitz metrics, one weak), equation, so we would expect only one jump condition.
} 


$$
T_{T O V}^{\mu \nu}=(\bar{\rho}+\bar{p}) \bar{u}_{T O V}^{\mu} \bar{u}_{T O V}^{\nu}+\bar{p} g^{\mu \nu},
$$

and the assumption that the TOV fluid is co-moving in barred coordinates,

$$
\bar{u}^{\mu}=\left[\begin{array}{c}
\sqrt{-A} \\
0
\end{array}\right]^{\mu}=\left[\begin{array}{c}
\sqrt{N-1} \\
0
\end{array}\right]^{\mu},
$$

we obtain

$$
T_{T O V}^{\mu \nu}=(\bar{\rho}+\bar{p})\left[\begin{array}{cc}
-A & 0 \\
0 & 0
\end{array}\right]^{\mu \nu}+\bar{p} A\left[\begin{array}{cc}
1 & 0 \\
0 & -\psi^{2}
\end{array}\right]^{\mu \nu} .
$$

Similarly,

$$
T_{F R W}^{\mu \nu}=(\rho+p) \bar{u}_{F R W}^{\mu} \bar{u}_{F R W}^{\nu}+p g^{\mu \nu},
$$

but in this case the assumption that the FRW fluid is co-moving in FRW unbarred coordinates $x^{\alpha}=(t, r)^{\alpha}$, implies that

$$
\bar{u}^{\mu}=\frac{\partial \bar{x}^{\mu}}{\partial x^{\alpha}} u^{\alpha},
$$

where

$$
u^{\alpha}=\left[\begin{array}{l}
1 \\
0
\end{array}\right]^{\alpha},
$$

gives the components of the FRW fluid velocity in standard FRW coordinates $(t, r)$.

Claim. When $k=0$, we have

$$
\frac{\partial \bar{x}^{\mu}}{\partial x^{\alpha}}=\left[\begin{array}{ll}
\sqrt{N} & R \\
\psi & \psi R \sqrt{N}
\end{array}\right]_{\alpha}^{\mu} .
$$

Proof of Claim. The coordinate mapping $\bar{r}=R r$ implies

$$
\begin{aligned}
& \frac{\partial \bar{x}^{0}}{\partial t}=\dot{R} r=H \bar{r}, \\
& \frac{\partial \bar{x}^{0}}{\partial r}=R,
\end{aligned}
$$

which verifies the the first row of (5.58) upon using (5.16), $H \bar{r}=\sqrt{N}$. Moreover, by (5.6)-(5.8),

$$
d \bar{t}=\psi\left(1-H^{2} \bar{r}^{2}\right) d t+\psi H \bar{r} d \bar{r} .
$$


Substituting

$$
d \bar{r}=d(R r)=\dot{R} r d t+R d r=H \bar{r} d t+R d r
$$

into (5.60) gives

$$
d \bar{t}=\psi(d t+H \bar{r} R d r)
$$

from which we can deduce the second row of (5.58), which verifies the Claim.

Continuing now from (5.56):

$$
\bar{u}^{\mu}=\left[\begin{array}{cc}
\sqrt{N} & R \\
\psi & \psi R \sqrt{N}
\end{array}\right]_{\alpha}^{\mu}\left[\begin{array}{l}
1 \\
0
\end{array}\right]^{\alpha}=\left[\begin{array}{c}
\sqrt{N} \\
\psi
\end{array}\right]^{\mu} .
$$

Using this in (5.55) gives

$$
T_{F R W}^{\mu \nu}=(\rho+p)\left[\begin{array}{cr}
N & \psi \sqrt{N} \\
\psi \sqrt{N} & \psi^{2}
\end{array}\right]^{\mu \nu}+p A\left[\begin{array}{cc}
1 & 0 \\
0 & -\psi^{2}
\end{array}\right]^{\mu \nu}
$$

Using (5.62) together with (5.55) gives

$$
\begin{aligned}
{[T]^{\mu \nu} } & =\left[T_{F R W}^{\mu \nu}-T_{T O V}^{\mu \nu}\right] \\
& =\left[\begin{array}{cr}
(\rho+p) N+(\bar{\rho}+p)(1-N) & \psi \sqrt{N}(\rho+p) \\
\psi \sqrt{N}(\rho+p) & \psi^{2}\{(\rho+\bar{p})+(p-\bar{p}) N\}
\end{array}\right],
\end{aligned}
$$

which is (5.49).

Finally, we use the Claim to verify (5.50) and (5.51) as follows. Let

$$
\phi(t, r) \equiv r-r(t)=0,
$$

where $r(t)$ denotes the shock surface in FRW $(t, r)$-coordinates. Then

$$
d \phi=-\dot{r} d t+d r=n_{0} d t+n_{1} d r,
$$

gives a covariant normal $n_{\alpha} d x^{\alpha}$ to the shock surface in $\mathbf{x}=(t, r)$ coordinates,

$$
\begin{aligned}
& n_{0}=-\dot{r}, \\
& n_{1}=1 .
\end{aligned}
$$

In $\overline{\mathbf{x}}=(\bar{r}, \bar{t})$-coordinates,

$$
\bar{n}_{\mu}=\frac{\partial x^{\alpha}}{\partial \bar{x}^{\mu}} n_{\alpha}
$$

where $\frac{\partial x^{\alpha}}{\partial \bar{x}^{\mu}}$ is the inverse of the matrix (5.58), 


$$
\frac{\partial x^{\alpha}}{\partial \bar{x}^{\mu}}=\frac{1}{\operatorname{det}\left|\frac{\partial \bar{x}^{\mu}}{\partial x^{\alpha}}\right|}\left[\begin{array}{cc}
\psi R \sqrt{N} & -R \\
-\psi & \sqrt{N}
\end{array}\right]_{\mu}^{\alpha} .
$$

Using (5.64) and (5.66) in (5.65) and neglecting the irrelevant factor $\frac{1}{\operatorname{det}\left|\frac{\partial \bar{x}^{\mu}}{\partial x^{\alpha}}\right|}$, we obtain, (to within a minus sign),

$$
\bar{n}_{\mu}=(\psi[\dot{r} R \sqrt{N}+1],-\dot{r} R-\sqrt{N})_{\mu},
$$

and substituting (5.21) in the form

$$
\dot{r}=\frac{\sqrt{N}}{R} \frac{p-\bar{p}}{\rho+\bar{p}}
$$

into (5.67), then gives the formulas for $\bar{n}_{\mu}$ given in (5.50), (5.51). The proof of Lemma 2 is complete.

We summarize the results in this section as follows: System (5.35)-(5.36) describes the TOV metrics that match a given $k=0 \mathrm{FRW}$ metric across a shock wave discontinuity when $A<0$, assuming that the conservation constraint (5.37) is satisfied. Solutions of these equations, together with the initial conditions in (5.39)-(5.43), determine weak solutions of the Einstein equations containing a shock wave interface across which the metric is only Lipschitz continuous, and such that conditions (i)-(iv) (after (5.2)) are satisfied. Since the resulting solutions are formally time-reversible, it still remains to impose a physically meaningful entropy condition. The entropy condition for shocks determines the time orientation of the solution, c.f. [12]. In the next section we construct a class of exact solutions of these equations which satisfy an entropy condition that agrees with the entropy condition of gas dynamics in the non-relativistic limit.

6. Exact Shock Wave Solutions When $A<0$. In this section we derive a class of exact solutions of equations (5.35)-(5.36) in the case when the FRW pressure is given by the equation of state

$$
p=\sigma \rho,
$$

where $\sigma$ is assumed to be constant,

$$
0<\sigma<1
$$

In this section, as an entropy condition, we impose the condition that the shock be compressive, a condition sufficient to choose the physically relevant stable shocks in classical gas dynamics, $[4,7,12]$. That is, we will require that the pressure and density be larger on the side of the shock that receives the mass flux. Exact solutions satisfying the equation of state (6.1) were constructed in [14] for the case $A>0$, so we can interpret the results here as an extension of the results in [14] to the case $A<0$. However, in [14], the conservation constraint led to a TOV equation of state also of 
the form (6.1), but in our case here, the TOV pressure is not so simple due to the fact that the conservation constraint (5.37) is of a different form.

Assuming (6.1), the solution to the FRW equations (2.7), (2.8) is given in Theorem 2 , equations (2.37)-(2.41), and we assume an expanding universe, $(\dot{R}>0)$, with initial conditions $R=0$ at $t=0$, and $R=1$, at $t=t_{0}$. The solution has one assignable constant which can be taken to be $t_{0}, \rho_{0}$ or $H_{0}$, since

$$
\begin{gathered}
\rho_{0}=\frac{4}{3 \kappa(1+\sigma)^{2}} \frac{1}{t_{0}^{2}}, \\
H_{0}=\left[\frac{3(1+\sigma)}{2}\right] \frac{1}{t_{0}},
\end{gathered}
$$

where we interpret $t_{0}$ as "present time" in the FRW metric ${ }^{13}$, and the relations (5.39)(5.43) hold.

We now solve the TOV shock equations (5.35)-(5.36), to obtain a shock wave solution that matches this FRW metric for all $R(t)$ in the interval between $R=0$ and $R=1$. Since (5.35) is coupled to (5.36) only through the function $\sigma$, our assumption that $\sigma$ is constant in the FRW metric implies that solutions of system (5.35)-(5.36) are determined by solutions of the scalar non-autonomous equation (5.35). Making the change of variable $S=1 / N$, (we do this to transform the "Big Bang" $N \rightarrow \infty$ over to rest point at $S \rightarrow 0$, c.f. [21]), equation (5.35) takes the form

$$
\frac{d u}{d S}=\left\{\frac{(1+u)}{2(1+3 u) S}\right\}\left\{\frac{(3 u-1)(\sigma-u)+6 u(1+u) S}{(\sigma-u)+(1+u) S}\right\}
$$

where $u=\bar{p} / \rho$. We now construct solutions of (6.5) that model the "Big Bang" as a localized explosion with an outgoing blast wave emanating from $\bar{r}=0$ at time $t=0$. Thus, motivated by classical gas dynamics, we seek solutions of (6.5) that satisfy the entropy conditions,

$$
\begin{aligned}
& 0<\bar{p}<p, \\
& 0<\bar{\rho}<\rho,
\end{aligned}
$$

and meet the physical bounds on the TOV equation of state

$$
0<\bar{p}<\bar{\rho} .
$$

Conditions (6.6), (6.7) for outgoing shock waves imply that the shock wave is compressive. The condition (6.8) implies that the TOV equation of state is physically

\footnotetext{
${ }^{13}$ Since we are assuming the idealized equation of state (6.1) for the FRW metric, the value of the Hubble constant at present time alone determines the solution. In the standard model of cosmology based on a $k=0$ FRW metric, [1], the solution is determined by two assignable constants, the Hubble constant $H_{0}$ and the background radiation temperature $T_{0},[1,21]$. Assuming (6.1), the freedom to assign $T_{0}$ is represented by the freedom to assign $\sigma$. Also, in the standard model the galaxies follow the particle paths of the matter field after the time when the pressure of the matter field is essentially zero. In the model (6.1), the matter is co-moving with respect to the radial coordinate $r$, and hence the particle paths follow $\dot{r}=0$. Loosely speaking, we refer to the motion along the particle paths as the motion of the galaxies in the cosmological interpretation of a general FRW metric. Thus we say that the shock wave is exploding outward through the galaxies when the shock wave satisfies $\dot{r}>0$,
} 
reasonable. Note that the conditions $N>1$ and $0<\bar{p}<p$ restrict the domain of (6.5) to the region $0<u<\sigma<1,0<S<1$. The following theorem shows that the inequalities (6.6), (6.7) and (6.8) can all be expressed in terms of $u$ and $S$ alone, and are all implied by a single inequality.

Theorem 5. Assume that

$$
0<u<\sigma
$$

$$
0<S<1
$$

and the conservation constraint (5.37) holds. Then the bounds (6.6)-(6.8) are all implied by the single condition

$$
S<\left(\frac{1-u}{1+u}\right)\left(\frac{\sigma-u}{\sigma+u}\right) \equiv E(u) .
$$

Proof of Theorem 5. Multiplying (6.9) through by $\rho>0$ gives (6.6). To verify that $\bar{\rho}<\rho$, write (5.37) in the form

$$
v=\frac{-\sigma S+a}{S+a}
$$

where

$$
a=\frac{\sigma-u}{1+u}>0
$$

and observe that $\bar{\rho}<\rho$ is equivalent to $v<1$, and by (6.12), $v<1$ if and only if $-\sigma<1$. To verify (6.8), observe that $\bar{p}<\bar{\rho}$ is equivalent to $\frac{v}{u}>1$, and by (6.12), this is equivalent to

$$
\frac{-\sigma S+a}{(S+a) u}>1
$$

which is easily seen to be equivalent to (6.11). Thus it remains only to verify $0<\bar{\rho}$. But observe that $\bar{\rho}>0$ is equivalent to $v>0$, and by (6.12), this is equivalent to

$$
S<\frac{1}{\sigma} \frac{\sigma-u}{1+u}
$$

which is implied by (6.11) because

$$
E(u)<\frac{1}{\sigma} \frac{\sigma-u}{1+u}
$$

The proof of the theorem is complete. 
6.1. Analysis of the equations. The purpose of this section is to prove the following theorem:

TheOREm 6. For every $\sigma, 0<\sigma<1$, there exists a unique solution $u_{\sigma}(S)$ of (6.5), such that (6.9) and (6.11) hold on the solution for all $S, 0<S<1$, and on this solution,

$$
\begin{aligned}
& 0<u_{\sigma}(S)<\bar{u}, \\
& \lim _{S \rightarrow 0} u_{\sigma}(S)=\bar{u},
\end{aligned}
$$

where

$$
\bar{u}=\operatorname{Min}\{\sigma, 1 / 3\},
$$

and

$$
\lim _{S \rightarrow 1} \bar{p}=0=\lim _{S \rightarrow 1} \bar{\rho} .
$$

Proof of Theorem 6. Solutions of (6.5) are determined by trajectories of the autonomous system

$$
\begin{aligned}
& S^{\prime}=2 S(1+3 u)\{(\sigma-u)+(1+u) S\}=F(S, u), \\
& u^{\prime}=(1+u)\{-(1-3 u)(\sigma-u)+6 u(1+u) S\}=G(S, u),
\end{aligned}
$$

which has two degenerate rest points, $S=0, u=\sigma$ and $S=0, u=1 / 3$, in the $(S, u)$ phase plane. Here "prime" denotes differentiation with respect to a parameter $\xi$, and we recover equation (6.5) by eliminating $\xi$ via $d u / d S=u^{\prime} / S^{\prime}$. Since $E(0)=1$, (c.f. (6.11)), the "initial condition" $u_{\sigma}(1)=0$ is a consequence of the entropy inequality (6.11), and thus the uniqueness of $u_{\sigma}(S)$ follows from uniqueness of solutions for system (6.19), (6.20) because $u=0, S=1$, is a regular initial condition for the system. Thus (6.18) follows from (5.34) and (5.37) because $\lim _{S \rightarrow 1} u_{\sigma}(S)=0$. It remains to prove the existence of the solution $u_{\sigma}(S)$ on $0<S<1$, and to establish (6.16).

Our analysis of these equations is based on the construction of invariant regions. Before we analyze the phase plane for system (6.19), (6.20), first note that $F(S, u)>0$ in the region $0 \leq u<\sigma, 0<S \leq 1$, and the isocline $G=0$ is given by

$$
S=\frac{(\sigma-u)(1 / 3-u)}{2 u(1+u)} \equiv h(u, \sigma) .
$$

Let $R_{\sigma}$ denote the region ${ }^{14}$

$$
R_{\sigma}=\{(S, u): 0 \leq u<\bar{u}, \quad 0<S \leq h(u, \sigma)\},
$$

\section{c.f. Figure 1.}

\footnotetext{
${ }^{14}$ We include the line $u=0$ in $R_{\sigma}$ in order that the initial point $u=0, S=1$ for the solution trajectory lies in $R_{\sigma}$. However, the entropy conditions (6.6), (6.8) do not hold at the transitional point $u=0, S=0$, the place where the solution $u_{\sigma}(S)$ continues naturally to the OS solution when $p<<1$.
} 


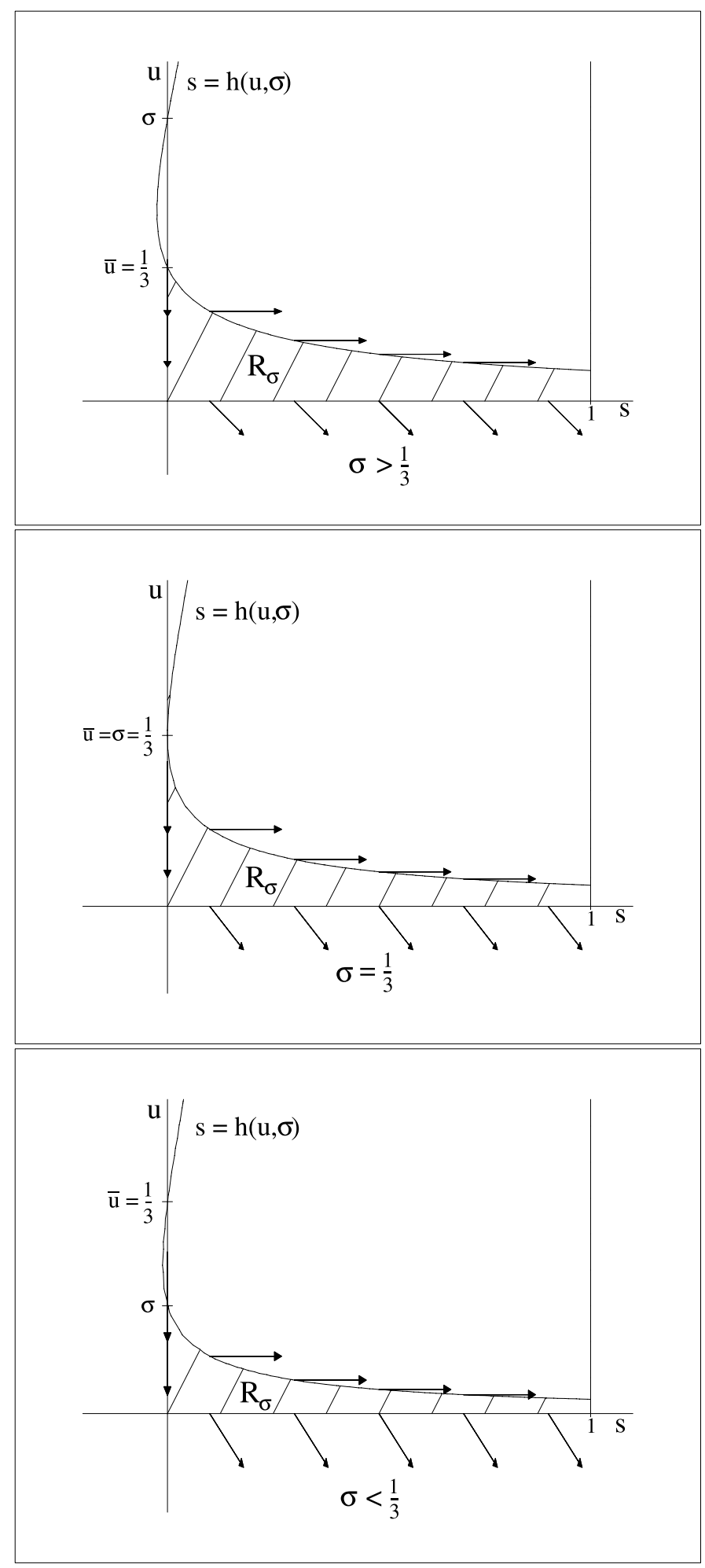

FIG. 1. The invariant region $R_{\sigma}$ 
LEMma 3. The region $R_{\sigma}$ is a negatively invariant region for system (6.19), (6.20) for each $\sigma$ between 0 and 1.

Proof. To show that $R_{\sigma}$ is negatively invariant, it suffices to show that the vector field $(F, G)$ points out of or tangent to $R_{\sigma}$ on the boundary of $R_{\sigma}$, as shown in Figure 1. Now the vector field $(F, G)$ is tangent to the axis $S=0$ because $S=0$ is a solution trajectory of system (6.19), (6.20). Since the boundary $u=0$ lies below the $G$-isocline, it follows that $G<0$ along the boundary $u=0$, and so the vector field $(F, G)$ points out of $R_{\sigma}$ along the lower boundary. Finally, the vector field points out of $R_{\sigma}$ along the upper boundary $S=h$ because, for each $\sigma, 0<\sigma<1, S=h$ is a $G$-isocline, $F$ is positive, and $S=h(u, \sigma)$ is a decreasing function of $u$ on $0 \leq u<\bar{u}$. Indeed, $S=h(u, \sigma)$ is decreasing on $0 \leq u<\bar{u}$ because, (using (6.21), and prime for $\left.\frac{d}{d u}\right)$,

$$
h^{\prime}=\frac{A^{\prime}}{B}-A\left(\frac{B^{\prime}}{B^{2}}\right) \leq 0
$$

where $h=\frac{A}{B}$ and

$$
\begin{aligned}
& A=(\sigma-u)(1 / 3-u), \\
& B=2 u(1+u),
\end{aligned}
$$

and we use the fact that $A \geq 0, A^{\prime} \leq 0, B \geq 0$, and $B^{\prime} \geq 0$ in $0 \leq u<\bar{u}$.

Now the existence of the orbit $u_{\sigma}(S)$ on the entire interval $0<S \leq 1$ is a consequence of the fact that $S=0, u=\bar{u}$ is the only rest point in the region $R_{\sigma}$. That is, the unique trajectory of system (6.19), (6.20) starting from initial point $(S, u)=(1,0) \in R_{\sigma}$, must tend to the unique rest point $(0, \bar{u})$ in backward time $\xi \rightarrow-\infty$. Moreover, since $G<0$ and $F>0$ in the interior of $R_{\sigma}$ it follows that $S$ and $u$ are monotone along the orbit, so the orbit defines the trajectory $u=u_{\sigma}(S)$, $0<S \leq 1$, as well as its inverse, $S=S_{\sigma}(u), 0 \leq u<\bar{u}$.

It remains only to verify that the entropy condition (6.11) holds all along the solution, and for this it suffices to show that

$$
\Delta(u) \equiv E(u)-S_{\sigma}(u) \geq 0,
$$

for all $u \in[0, \bar{u})$. But

$$
\Delta(0)=0
$$

because $E(0)=1=S_{\sigma}(0)$, and

$$
\Delta(\bar{u}) \geq 0
$$

because $E(\bar{u}) \geq 0$ and $S_{\sigma}(\bar{u})=0$. Thus to verify that $\Delta(u)>0$ for $0 \leq u<\bar{u}$, it suffices only to show that $\Delta(u)>0$ in a deleted neighborhood of $u=0$, and that $\Delta^{\prime}(u)=0$ has at most one root in $0 \leq u<\bar{u}$. But differentiating (6.11) gives

$$
E^{\prime}(u)=-2(1+\sigma) \frac{\sigma-u^{2}}{(\sigma+u)^{2}(1+u)^{2}} .
$$

Using this and (6.5) at $u=0$ gives 


$$
E^{\prime}(0)=-2 \frac{1+\sigma}{\sigma}=s_{\sigma}^{\prime}(0)
$$

and

$$
E^{\prime \prime}(0)=4 \frac{(1+\sigma)^{2}}{\sigma^{2}}>-6 \frac{(1+\sigma)^{2}}{\sigma^{2}}=s_{\sigma}^{\prime \prime}(0),
$$

which implies that $\Delta^{\prime}(0)=0, \Delta^{\prime \prime}(0)>0$, so $\Delta(u)>0$, near $u=0$. Moreover, by (6.11) and (6.5) we also see that $\Delta^{\prime}(u)=0$ if and only if

$$
\begin{aligned}
& S^{2}\left\{(\sigma+u)^{2}(1+u)^{3}(1+3 u)\right\}_{a} \\
& \quad+S\left\{(\sigma-u)(\sigma+u)^{2}(1+u)^{2}(1+3 u)+6 u(1+\sigma)(1+u)^{2}\left(\sigma-u^{2}\right)\right\}_{b} \\
& \quad+\left\{(3 u-1)(1+\sigma)(\sigma-u)(1+u)\left(\sigma-u^{2}\right)\right\}_{c}=0 .
\end{aligned}
$$

But $\{\cdot\}_{a}>0,\{\cdot\}_{b}>0$, and $\{\cdot\}_{c}<0$ for $0<u<\bar{u}$ because $\bar{u}=\operatorname{Min}\{\sigma, 1 / 3\}$, and thus the quadratic (6.27) has exactly one positive root. We conclude that (6.24) holds. Finally, to verify (6.18), we note that $u \rightarrow 0$ as $S \rightarrow 1$, so $\bar{p} \rightarrow 0$. The fact that $\lim _{S \rightarrow 1} \bar{\rho}=0$ follows directly from (5.37). The proof of Theorem 6 is complete.

Theorem 6 implies that the entropy conditions (6.6), (6.7) pick out the unique solution of system (6.5) emanating from the initial point $S=1, u \equiv \frac{\bar{p}}{\rho}=0=\bar{p}$.

6.2. The shock speed. In this section we estimate the shock speed $s_{\sigma}(S) \equiv$ $s\left(u_{\sigma}(S)\right)$, the speed of the shock relative to an observer fixed relative to the FRW fluid, along the solutions $u_{\sigma}(S)$ of Theorem $6,0<S<1,0<u_{\sigma}(S)<\sigma$.

TheOREM 7. Let $0<\sigma<1$. Then the shock wave is everywhere subluminous, that is,

$$
\left|s_{\sigma}(S)\right|<1
$$

for all $0<S \leq 1$, if and only if

$$
\sigma \leq 1 / 3
$$

Proof. Formula (5.38) gives

$$
s_{\sigma}(S)=R \dot{r}=\frac{1}{\sqrt{S}}\left(\frac{\sigma-u}{1+u}\right),
$$

and thus since $\lim _{S \rightarrow 0} u_{\sigma}(S)=\bar{u}=\operatorname{Min}\{\sigma, 1 / 3\}$, it follows at once that $\lim _{S \rightarrow 0} s_{\sigma}(S)=\infty$ if $\sigma>1 / 3$. Since $\lim _{S \rightarrow 0} u_{\sigma}(S)=\sigma$ when $\sigma \leq 1 / 3$, we conclude from (6.30) that the precise value of the shock speed $s_{\sigma}$ in the limit $S \rightarrow 0$ depends on the asymptotic behavior of the solution $u_{\sigma}(S)$ as $S \rightarrow 0$. This is addressed in the next subsection.

To verify (6.28), (6.29), we first prove the following lemma.

Lemma 4. Assume that $0<\sigma \leq 1 / 3$, and let $S=Q_{a}(u) \equiv Q(u)$ be defined by 


$$
Q_{a}(u)=a^{2} \frac{(\sigma-u)^{2}}{(1+u)^{2}}
$$

Then the region

$$
\mathcal{Q}_{\sigma}^{a} \equiv\left\{(S, u): S \geq Q_{a}(u)\right\} \cap R_{\sigma}
$$

is a negatively invariant region for solutions of system (6.19), (6.20), so long as

$$
a^{2} \leq \frac{1}{3 \sigma}
$$

Moreover, for $\sigma=1 / 3$ and sufficiently small $\epsilon>0$, there exists a $\delta>0$, such that if

$$
a^{2} \leq \frac{1}{1-\delta}
$$

then solutions of (6.19), (6.20), starting in the region

$$
\mathcal{Q}_{1 / 3}^{a}(\epsilon) \equiv \mathcal{Q}_{1 / 3}^{a} \cap\{(S, u): 0 \leq u<1 / 3-\epsilon\}
$$

can only leave the region $\mathcal{Q}_{1 / 3}^{a}(\epsilon)$ through the boundary $u=1 / 3-\epsilon$.

Note first that, assuming $\sigma \leq 1 / 3$, the condition $a^{2} \leq \frac{1}{2 \sigma}$ guarantees that $Q_{a}(u)<$ $h(u)$ in the region $0 \leq u<\sigma, 0<S \leq 1$ because by $(6.21), Q_{a}(u)<h(u)$ is equivalent to

$$
a^{2}<\left(\frac{1 / 3-u}{\sigma-u}\right) \frac{(1+u)}{2 u}
$$

and since

$$
\frac{1}{2 \sigma}<\left(\frac{1 / 3-u}{\sigma-u}\right) \frac{(1+u)}{2 u}
$$

$a^{2} \leq \frac{1}{2 \sigma}$ guarantees (6.36) in $0 \leq u<\sigma, 0<S \leq 1$. It follows that when $a^{2} \leq \frac{1}{2 \sigma}$, the region $\mathcal{Q}_{\sigma}^{a}$ is the region in the $(S, u)$-plane that lies above the curve $S=Q_{a}(u)$, above the curve $u=0$, and below the isocline $S=h(u)$. Moreover, when $a^{2} \leq \frac{1}{2 \sigma}$, the initial point $u=0, S=1$ for solution trajectory $u_{\sigma}(S)$ lies within the region $\mathcal{Q}_{\sigma}^{a}$ because $Q_{a}(0)=a^{2} \sigma^{2}<1$.

Before proving the lemma, we first use Lemma 4 to complete the proof of Theorem 7 by verifying (6.28). Since the initial condition $S=1, u=0$ lies within the region $\mathcal{Q}_{\sigma}^{a}$, we can estimate the shock speed all along the orbit $u_{\sigma}(S)$ by

$$
s_{\sigma}(u)=\frac{1}{\sqrt{S}}\left(\frac{\sigma-u}{1+u}\right) \leq \frac{1}{a}\left(\frac{1+u}{\sigma-u}\right)\left(\frac{\sigma-u}{1+u}\right)=\frac{1}{a},
$$

and so taking $a=\frac{1}{\sqrt{3 \sigma}}<\frac{1}{\sqrt{2 \sigma}}$, (optimal for $(6.33)$ ), we obtain 


$$
s_{\sigma}(u) \leq \sqrt{3 \sigma}<1,
$$

for all $0<\sigma<1 / 3,0 \leq u \leq \sigma$. This verifies (6.28) for $0<\sigma<1 / 3$. However, (6.39) does not provide a strict inequality if $\sigma=1 / 3$.

To obtain the strict inequality (6.28) in the case $\sigma=1 / 3$, we use that the initial condition $S=1, u=0$ also lies in $\mathcal{Q}_{1 / 3}^{a}(\epsilon)$ for $a=\frac{1}{\sqrt{1-\delta}}$, so if we assume $u_{1 / 3}(S) \leq$ $1 / 3-\epsilon$, then (6.34), (6.35) imply that the shock speed in the region $\mathcal{Q}_{1 / 3}^{a}(\epsilon)$ can be estimated by

$$
s_{1 / 3}(u) \leq \frac{1}{a}=\sqrt{1-\delta}<1,
$$

when $\sigma=1 / 3$. Since $\epsilon$ is arbitrary, we conclude that (6.28) holds for all $0<\sigma \leq 1 / 3$, $0<S \leq 1$.

The proof of Theorem 7 is complete, once we give the

Proof of Lemma 4. Assume (6.29). To show that $\mathcal{Q}_{\sigma}^{a}$ is negatively invariant, it suffices to prove that the vector field $(F, G)$ restricted to the curve $S=Q_{a}(u)$, always points into the region below the curve. That is, it suffices to prove that $\left|Q^{\prime}(u)\right| \geq\left|\frac{d S}{d u}\right|$ on $S=Q_{a}(u) \equiv Q$, where $\frac{d S}{d u}$ is given in (6.5). Thus our condition is

$$
\left|Q^{\prime}\right|=2 a^{2} \frac{(1+\sigma)(\sigma-u)}{(1+u)^{3}} \geq \frac{2(1+3 u) Q[(\sigma-u)+(1+u) Q]}{(1+u)|(3 u-1)(\sigma-u)+6 u(1+u) Q|} .
$$

After some algebra, this reduces to

$$
\frac{(1+\sigma)}{(\sigma-u)(1+3 u)} \geq \frac{(1+u)+(\sigma-u) a^{2}}{\left|(1-3 u)(1+u)-6 u(\sigma-u) a^{2}\right|} .
$$

But the term inside the absolute value is positive so long as $a \leq \frac{1}{2 \sigma}$ because then

$$
a^{2} \leq \frac{1}{2 \sigma} \leq\left(\frac{1-3 u}{\sigma-u}\right) \frac{1+u}{6 u}
$$

as a result of (6.37).

Therefore, using that the term in the absolute value in (6.41) is positive, we can solve (6.41) for $a^{2}$ to obtain the following condition equivalent to the condition that $S \geq Q(u)$ is negatively invariant:

$$
\begin{aligned}
a^{2} & \leq \frac{(1+u)[(1+\sigma)(1-3 u)-(1+3 u)(\sigma-u)]}{(1+3 u)(\sigma-u)^{2}+(1+\sigma) 6 u(\sigma-u)} . \\
& =\frac{(1+u)\left[(1+\sigma) \frac{(1-3 u)}{(\sigma-u)}-(1+3 u)\right]}{(1+3 u)(\sigma-u)+6(1+\sigma) u}
\end{aligned}
$$

But using that

$$
\frac{1-3 u}{\sigma-u} \geq 3
$$


it follows that the inequality

$$
a^{2} \leq \frac{(1+u)[2+3(\sigma-u)]}{(1+3 u)(\sigma-u)+6(1+\sigma) u} \equiv \phi_{\sigma}(u),
$$

guarantees (6.43) all along the curve $S=Q_{a}(u)$. But $\phi_{\sigma}^{\prime}(u)<0$ for $0 \leq u \leq \sigma \leq 1 / 3$ because $\phi_{\sigma}(u)=\frac{A}{B}$ where

$$
\begin{gathered}
A=(1+u)[2+3(\sigma-u)], \\
B=(1+3 u)(\sigma-u)+6(1+\sigma) u,
\end{gathered}
$$

and

$$
B A^{\prime}-A B^{\prime}<0
$$

Therefore,

$$
\phi_{\sigma}(u) \geq \phi_{\sigma}(\sigma)=\frac{1}{3 \sigma}
$$

From this we conclude that if (6.33) holds, that is, if $a^{2} \leq \frac{1}{3 \sigma}<\frac{1}{2 \sigma}$, then (6.44) holds, and hence $\mathcal{Q}_{\sigma}^{a}$ is an invariant region for all $0<\sigma \leq 1 / 3$, as claimed. It remains to verify (6.34) and (6.35) in the case $\sigma=1 / 3$.

So assume $\sigma=1 / 3$. Then from (6.44), we have

$$
\phi_{1 / 3}(u)=\frac{(1+u)[2+3(1 / 3-u)]}{(1+3 u)(1 / 3-u)+6(1+1 / 3) u} .
$$

But since, $\phi_{1 / 3}^{\prime}(u)<0$ for $0 \leq u \leq 1 / 3$, it follows that for every $\epsilon>0$ there exists a $\delta>0$ such that

$$
\phi_{1 / 3}(u)>\frac{1}{1-\delta}
$$

in the region $0 \leq u \leq 1 / 3-\epsilon$. Thus, if we choose $a^{2} \leq \frac{1}{1-\delta}$, then (6.44) holds, and so $S \geq Q_{1 / 3}(u)$ is an invariant region for $0 \leq u \leq 1 / 3-\epsilon$. It follows that (6.34) implies that orbits can only leave the region $\mathcal{Q}_{1 / 3}^{a}(\epsilon)$ through the line $u=1 / 3-\epsilon$, as claimed. The proof of Lemma 4 is complete.

6.3. The shock speed at the Big Bang. In this section we calculate the shock speed $s_{\sigma}(S)$ along the orbit $u=u_{\sigma}(S)$ in the limit $S \rightarrow 0$, the instant of the Big Bang. In Theorem 7 we showed that $\lim _{S \rightarrow 0} s_{\sigma}=\infty$ if $\sigma>1 / 3$, and the following theorem asserts that $\lim _{S \rightarrow 0} s_{\sigma}=0$ for $\sigma<1 / 3$, but that $\lim _{S \rightarrow 0} s_{\sigma}=1$ for the special value $\sigma=1 / 3$. In particular, this confirms that the estimates of Theorem 7 are sharp.

TheOREM 8. Let $0<\sigma \leq 1 / 3$, and let $s_{\sigma}(S) \equiv s_{\sigma}\left(u_{\sigma}(S)\right)$ denote the shock speed along the solution $u=u_{\sigma}(S)$, given in (6.30). Then if $\sigma>1 / 3$,

$$
\lim _{S \rightarrow 0} s_{\sigma}(S)=\infty
$$

if $\sigma<1 / 3$

$$
\lim _{S \rightarrow 0} s_{\sigma}(S)=0
$$


and if $\sigma=1 / 3$,

$$
\lim _{S \rightarrow 0} s_{\sigma}(S)=1
$$

Proof. By (6.30), the shock speed is given by

$$
s_{\sigma}(S)=\frac{1}{\sqrt{S}}\left(\frac{\sigma-u_{\sigma}(S)}{1+u_{\sigma}(S)}\right)
$$

from which (6.45) is evident. To verify (6.46), we show that when $0<\sigma<1 / 3$, and $a=\frac{1}{\sqrt{3 \sigma}}$, there exists $m>0$ such that

$$
(1,0) \in R_{\sigma}^{m} \equiv\{(S, u): S \geq m(\sigma-u)\} \cap \mathcal{Q}_{\sigma}^{a},
$$

and that the region $R_{\sigma}^{m}$ is negatively invariant for solutions of system (6.19), c.f. (6.32). If $R_{\sigma}^{m}$ is negatively invariant and contains the initial point $(S, u)=(1,0)$, then $u_{\sigma}(S) \geq \sigma-\frac{1}{m} S$ in a neighborhood of $u=\sigma$, which implies (6.46) because then we can use (6.48) to conclude

$$
0 \leq \lim _{S \rightarrow 0} s_{\sigma}(S) \leq \lim _{S \rightarrow 0} \frac{1}{\sqrt{S}}\left(\frac{\frac{1}{m} S}{1+u_{\sigma}(S)}\right)=0 .
$$

To see that $R_{\sigma}^{m}$ is negatively invariant for some $m>0$, assume first that

$$
m \leq h^{\prime}(\sigma)=\frac{1-3 \sigma}{6 \sigma(1+\sigma)}
$$

c.f. (6.21). This implies that $m(\sigma-u) \leq h(u)$ in the region $0 \leq u<\sigma, 0<S \leq 1$. Thus when $m \leq h^{\prime}(\sigma)$, the region $R_{\sigma}^{m}$ consists of the set of all points in $\mathcal{Q}_{\sigma}^{a}$ that lie above the curve $S=m(\sigma-u)$ and below the curve $S=h(u)$. It is also easily verified that the initial condition $S=1, u=0$ lies in $R_{\sigma}^{m}$ when $m \leq h^{\prime}(\sigma)$.

To find a value of $m \leq h^{\prime}(\sigma)$ for which $R_{\sigma}^{m}$ is negatively invariant, we mimic the proof of Lemma 4 using $L \equiv L(u)=m(\sigma-u)$ in place of $Q_{\sigma}(u)$. That is, $R_{\sigma}^{m}$ is negatively invariant if the vector field $(F, G)$ points into the region below $S=L(u)$, all along $S=L(u)$, which is to say that $\left|L^{\prime}(u)\right| \geq\left|\frac{d S}{d u}\right|$ on $S=L(u)$, where $\frac{d S}{d u}$ is given in (6.5). Since we have already verified that $\mathcal{Q}_{\sigma}^{a}$ is negatively invariant for $a=\frac{1}{\sqrt{3 \sigma}}$, we actually need only verify the negative invariance of the curve $S=L(u)$ for values $\bar{u} \leq u \leq \sigma$, where $\bar{u}$ is the value of $u$ at the point of intersection $(\bar{S}, \bar{u})$ of the line $S=L(u)$ and the quadratic $S=Q_{a}(u)=\frac{1}{3 \sigma} \frac{(\sigma-u)^{2}}{(1+u)^{2}}$, c.f. (6.31). Since $L$ is linear and $Q_{a}$ is quadratic at $(0, \sigma)$, it follows that $\bar{u} \rightarrow \sigma$ as $m \rightarrow 0$. Thus our condition is

$$
\left|L^{\prime}\right|=m \geq \frac{2 L(1+3 u)[L(1+u)+(\sigma-u)]}{(1+u)|(\sigma-u)(3 u-1)+6 u(1+u) L|},
$$

for $\bar{u} \leq u \leq \sigma$. After some algebra, this reduces to

$$
1 \geq \frac{2(\sigma-u)(1+3 u)[m(1+u)+1]}{|(1+u)|(3 u-1)+6 u(1+u) m \mid} .
$$

But the term inside the absolute value is negative because 


$$
m \leq \frac{1-3 u}{6 u(1+u)}
$$

a consequence of the fact that we are assuming $m \leq h^{\prime}(\sigma)$. Therefore, we can solve (6.50) for $m$ to obtain the following condition equivalent to the condition that $S \geq$ $L(u)$ is negatively invariant:

$$
m \leq \frac{1-3 u-2(1+3 u)(\sigma-u)}{(1+u)[2(1+3 u)(\sigma-u)+6 u]}=\psi_{\sigma}(u) .
$$

Now assuming $\sigma<\frac{1}{3}$ is fixed, it follows that $\psi=A / B$, where

$$
B=(1+u)[2(1+3 u)(\sigma-u)+6 u] \leq 2(1+\sigma)\left(7 \sigma+3 \sigma^{2}\right),
$$

and

$$
A=1-3 u-2(1+3 u)(\sigma-u) .
$$

Therefore, to verify (6.52) for $\bar{u} \leq u \leq \sigma$ for some $m>0$, it suffices only to show that for $m$ sufficiently small, $A=1-3 u-2(1+3 u)(\sigma-u)$ is bounded uniformly away from zero for all $u \in[\bar{u}, \sigma]$. But $1-3 \sigma=\epsilon>0$, so $\lim _{m \rightarrow 0} \bar{u}=\sigma$ implies that there exists an $\delta>0$ such that $m<\delta$ guarantees that $2(1+3 u)(\sigma-u)<\epsilon / 2$ for all $u \in[\bar{u}, \sigma]$. In this case, $A=1-3 u-2(1+3 u)(\sigma-u) \geq \frac{\epsilon}{2}$, and hence by (6.53),

$$
\psi_{\sigma}(u) \geq \frac{\epsilon}{4(1+\sigma)\left(7 \sigma+3 \sigma^{2}\right)} \equiv \delta_{1} .
$$

It follows from (6.52) that if we choose $m=\operatorname{Min}\left\{\delta, \delta_{1}\right\}$, Then for fixed $\sigma<\frac{1}{3}$, it follows that

$$
m \leq \psi_{\sigma}(u)
$$

for all $u \in[\bar{u}, \sigma]$, and hence that $R_{\sigma}^{m}$ is negatively invariant. Thus the proof of (6.46) is complete, and it remains only to consider the case $\sigma=1 / 3$.

In the case $\sigma=1 / 3, R_{\sigma}^{m}$ is not negatively invariant for any $m>0$, and to evaluate $\lim _{S \rightarrow 0} s_{\sigma}(S)$ in this case we need the asymptotics of the solution $u_{1 / 3}(S)$ near $S=0$. This is given in the following lemma:

Lemma 5. Assume $\sigma=1 / 3$. Then

$$
u_{\sigma}(S) \sim 1 / 3-m_{*} \sqrt{S}, \quad \text { as } S \rightarrow 0,
$$

where

$$
m_{*}=\frac{4}{3}
$$

Lemma 5 implies (6.47) of Theorem 8, (the case $\sigma=1 / 3$ ), because using $(6.56)$ in (6.30) we obtain,

$$
\begin{aligned}
s_{\sigma}(S) & =\frac{1}{\sqrt{S}}\left(\frac{\sigma-u_{\sigma}(S)}{1+u_{\sigma}(S)}\right) \\
& =\frac{1}{\sqrt{S}}\left(\frac{m_{*} \sqrt{S}}{1+u_{\sigma}(S)}\right) \rightarrow \frac{\frac{4}{3}}{1+\frac{1}{3}}=1 .
\end{aligned}
$$


Note that (6.56) confirms that the orbit $u_{1 / 3}(S)$ comes into the rest point $S=0$, $u=1 / 3$, asymptotically like the negatively invariant curve $S=Q_{1}(u)=\left(\frac{3}{4}\right)^{2}\left(\frac{1}{3}-u\right)^{2}$, c.f. $(6.31)$.

Proof of Lemma 5. Assume $\sigma=1 / 3$. To verify (6.56), write

$$
u_{\sigma}(S)=1 / 3+\phi(S), \quad 0<S \leq 1 .
$$

We find the equation that $\phi$ satisfies asymptotically as $S \rightarrow 0$. (Note that $\phi(0)=0$ because $u_{\sigma}(S)$ tends to the rest point $(0,1 / 3)$ as $S \rightarrow 0$.) Putting (6.59) into (6.5) we obtain

$$
S \phi^{\prime}(S)=\frac{\left(\frac{4}{3}+\phi\right)\left[-3 \phi^{2}+(2+6 \phi)\left(\frac{4}{3}+\phi\right) S\right]}{2(2+3 \phi)\left[-\phi+\left(\frac{4}{3}+\phi\right) S\right]} .
$$

But by Lemma 4 , we know that $\mathcal{Q}_{1 / 3}^{a}$ is negatively invariant for $a=1$, and from this it follows that $u_{1 / 3}(S)$ is squeezed below the $G$-isocline and above the curve $u=Q^{-1}(S)=1 / 3-\sqrt{S}$ as $S \rightarrow 0$. It follows that $\left(1 / 3-u_{1 / 3}(S)\right)$ is order $S^{1 / 2}$ as $S \rightarrow 0$. Using this in $(6.60)$ gives

$$
S \phi^{\prime}(S) \sim \frac{\left(\frac{4}{3}\right)\left[-3 \phi^{2}+\frac{8}{3} S\right]}{2(2)[-\phi]},
$$

where $\sim$ means to leading order in $S$ as $S \rightarrow 0$. Thus, to leading order in $S$, equation (6.61) takes the asymptotic form

$$
S \phi^{\prime}=\phi-\frac{8}{9} \frac{S}{\phi}
$$

which we can write in the form

$$
\left(\phi^{2}\right)^{\prime}=\frac{\alpha \phi^{2}}{S}-\beta
$$

which is linear in $v=\phi^{2}$ with

$$
\alpha=2,
$$

and

$$
\beta=16 / 9
$$

But (6.63) has the general solution

$$
v=k S^{\alpha}-\frac{\beta}{1-\alpha} S,
$$

where $k$ is a constant. Since $\alpha>1$, the first term in (6.66) is higher order in $S$, and thus we have shown that to leading order in $S$, equation (6.62) has the unique solution

$$
\phi(S)=m_{*} \sqrt{S},
$$


where

$$
m_{*}=\sqrt{\frac{\beta}{\alpha-1}}=4 / 3 .
$$

This agrees with (6.56) and (6.57). Thus the proof of Lemma 4 will be complete once we prove the following lemma.

Lemma 6. Assume $\sigma=1 / 3$. Then every solution $u(S)$ of (6.5) that enters the rest point $S=0, u=1 / 3$ from inside the invariant region $\mathcal{Q}_{1 / 3}^{a}, a=1$, must satisfy

$$
u(S) \sim 1 / 3-\frac{4}{3} \sqrt{S}, \quad \text { as } S \rightarrow 0 .
$$

Proof. By the derivation of (6.62), we know that

$$
u(S)=1 / 3+\hat{\phi}(S),
$$

where $\hat{\phi}$ satisfies

$$
\left(\hat{\phi}^{2}\right)^{\prime}=\alpha \frac{\hat{\phi}^{2}}{S}-\beta-\epsilon(S),
$$

for some function, $\epsilon(S) \rightarrow 0$ as $S \rightarrow 0$. Thus it suffices to show that $\lim _{S \rightarrow 0}\left|\frac{\hat{\phi}^{2}(S)-\phi^{2}(S)}{S}\right|=0$. But (6.70) is linear and has the general solution

$$
\hat{\phi}^{2}(S)=K S^{\alpha}+S^{\alpha} \int_{S}^{S_{0}}\{\beta+\epsilon(t)\} t^{-\alpha} d t
$$

for some constants $K$ and $S_{0}$. Moreover, $\phi^{2}(S)=m_{*}^{2} S$ also satisfies

$$
\phi^{2}(S)=\frac{\beta}{\alpha-1} S_{0}^{-\alpha+1} S^{\alpha}+S^{\alpha} \int_{S}^{S_{0}} \beta t^{-\alpha} d t,
$$

as one easily sees by integration, and consistent with the fact that $\phi$ solves (6.70) with $\epsilon=0$. Thus

$$
\begin{aligned}
\hat{\phi}^{2}-\phi^{2} & =-\frac{\beta}{\alpha-1} S_{0}^{-\alpha+1} S^{\alpha}+S^{\alpha} \int_{S}^{S_{0}} \epsilon(t) t^{-\alpha} d t+K S^{\alpha} \\
& =-\frac{\beta}{\alpha-1} S_{0}^{-\alpha+1} S^{\alpha}+\epsilon_{*} S^{\alpha} \int_{S}^{S_{0}} t^{-\alpha} d t+K S^{\alpha} \\
& =-\frac{\beta}{\alpha-1} S_{0}^{-\alpha+1} S^{\alpha}+\epsilon_{*}\left(\frac{1}{1-\alpha}\right)\left[S_{0}^{-\alpha+1} S^{\alpha}-S\right]+K S^{\alpha},
\end{aligned}
$$

where we have applied the mean value theorem for integrals with $\epsilon_{*}=\epsilon\left(S_{*}\right)$ for some $S_{*} \in\left(S, S_{0}\right)$. Now if we choose $S_{0}=S^{a}$ for some $0<a<1$, then every term in (6.73) is higher order than $S$. That is,

$$
\left|\hat{\phi}^{2}-\phi^{2}\right|(S) \leq\left[\frac{\beta+\epsilon_{*}}{\alpha-1} S^{(\alpha-1)(1-a)}+|K| S^{\alpha}+\frac{\epsilon_{*}}{\alpha-1}\right] S,
$$


from which we conclude that

$$
\lim _{S \rightarrow 0}\left|\frac{\hat{\phi}^{2}(S)-\phi^{2}(S)}{S}\right|=0,
$$

as claimed. This completes the proof of Lemma 6 , and thus Lemma 5 as well.

6.4. The asymptotics for $S \geq 1$. Equation (6.18) implies that $v \equiv \frac{\bar{\rho}}{\rho}=0=\bar{\rho}$ at $S=1$. Thus, the TOV metric inside the Black Hole continues to the empty space Schwarzschild metric at $u=0, v=0, S=1$, an event horizon for the outer TOV metric in light of the fact that at $S=1, N=\frac{2 M}{\bar{r}}=1$, c.f. (5.17). It follows that if the FRW density $\rho$ is small at $S=1$, then it makes sense in cosmology to approximate the FRW solution with $p=0$ for all times $S \geq 1$. Assuming this, Theorem 6 implies that for each $0<\sigma<1$, the unique solution $u_{\sigma}(S)$ continues to the zero pressure, $k=0$, OS solution at $S=N=1$, the moment when the shock wave lies exactly one Hubble length from the FRW center. That is, at $S=1$, the shock wave emerges from the White Hole event horizon of an ambient Schwarzschild metric as an outward propagating contact discontinuity that bounds a finite FRW mass, this being exactly equal to the total mass of the ambiant Schwarzschild metric into which it propagates. Thereafter the interface continues out to infinity along a geodesic of the Schwarzschild metric outside the Black Hole.

We conclude that the OS solution gives the large time asymptotics of this new class of shock wave solutions that evolve inside of a Black Hole - and thus the explosion that begins at the Big Bang eventually settles down to a localized expansion that looks something like a giant supernovae, but on an enormous scale. .

7. Estimates for the Shock Position. In this section we use the invariant region $\mathcal{Q}_{\sigma}^{a}$, (c.f. (6.32)), to estimate the shock position $\bar{r}_{0}$ at present time in terms of its position at the instant of the Big Bang $S \equiv 1 / N=0$. Using this, we finish by estimating the time at which the shock wave first leaves the Black Hole in terms of the time at which the shock wave first becomes visible to an observer at the FRW center.

Since the physical shock position at $S=0$ is $\bar{r}=0$, we begin by estimating $\bar{r}_{0}=r_{0}, R\left(t_{0}\right)=1$, in terms of $r_{*}$, the value of the radial FRW coordinate at the instant of the Big Bang. In particular, the analysis shows that $r_{\sigma}(S)$ has a limit $r_{*} \equiv r_{*}(\sigma)$ as $S \rightarrow 0$, for any solution $\left(r_{\sigma}(S), u_{\sigma}(S)\right)$ of system (5.35),(5.36). So assume the FRW solution for the equation of state $p=\sigma \rho, 0<\sigma \leq 1 / 3$, is given, together with the shock trajectory $u_{\sigma}(S)$, that solves equation (5.35). There then remains the one equation (5.36), leaving one free initial condition to impose. The next lemma gives an equivalent form of equation (5.36) in terms of the FRW variable $r$.

Lemma 7. Equation (5.36) is equivalent to

$$
\frac{d r}{d S}=\frac{\sigma-u}{(1+\sigma)(1+3 u)} \frac{r}{S},
$$

for all $0<S \leq 1$.

Proof. Starting with (5.36), we can write 


$$
\frac{d \bar{r}}{d S}=\frac{d \bar{r}}{d N} \frac{d N}{d S}=\frac{1}{(1+3 u)} \frac{\bar{r}}{S}
$$

Also,

$$
\frac{d r}{d S}=\frac{d\left(\frac{\bar{r}}{R}\right)}{d S}=\frac{1}{R} \frac{d \bar{r}}{d S}-\frac{\bar{r}}{R^{2}} \frac{d R}{d S}
$$

so using (7.2) we have

$$
\frac{d r}{d S}=\left(\frac{1}{1+3 u}\right) \frac{r}{S}-\frac{r}{R} \frac{d R}{d S}
$$

So now we need only compute $d R / d S$. For this, we use $(2.38),(2.39)$ to obtain

$$
H=H_{0} R^{-\frac{3(1+\sigma)}{2}},
$$

and from (5.16),

$$
H^{2} \bar{r}^{2}=N=1 / S
$$

These imply

$$
R=\left(S H_{0}^{2} \bar{r}^{2}\right)^{\frac{1}{3(1+\sigma)}}
$$

Using (7.4) together with (7.2) gives

$$
\frac{d R}{d S}=\frac{(1+u)}{(1+\sigma)(1+3 u)}\left(H_{0}^{2} \bar{r}^{2}\right)^{\frac{1}{3(1+\sigma)}} S^{-\frac{(2+3 \sigma)}{3(1+\sigma)}} .
$$

From (7.4) we get

$$
H_{0}^{2} \bar{r}^{2}=\frac{R^{3(1+\sigma)}}{S},
$$

and using this in (7.5) gives

$$
\frac{d R}{d S}=\frac{(1+u)}{(1+\sigma)(1+3 u)} \frac{R}{S} .
$$

Use this in (7.3) to get (7.1).

We now use (7.1) to estimate $r_{0}$ in terms of $r_{*}$, assuming that the shock position $\bar{r}_{0}=r_{0}=\sqrt{N_{0}} / H_{0}$ at present time lies beyond one Hubble length. Then $N_{0}>1$, and we have 


$$
S_{0}=1 / N_{0}=\frac{1}{H_{0} \bar{r}_{0}^{2}}<1
$$

c.f. (2.7), (5.17). Then integrating equation (7.1) we obtain,

$$
r_{0}=r_{*} e^{\int_{0}^{S_{0}}\left(\frac{\sigma-u}{1+3 u}\right) \frac{1}{(1+\sigma) S} d S},
$$

where $u$ denotes the function of $S$ given by the trajectory $u=u_{\sigma}(S)$ ) of (5.35), $u_{\sigma}(0)=\sigma \leq 1 / 3, u_{\sigma}(1)=0, u_{\sigma}^{\prime}(S)<0,0 \leq S \leq 1$. We can now use the invariant region $\mathcal{Q}_{\sigma}^{a}$ to estimate $\sigma-u_{\sigma}(S)$ for $0<S \leq 1$. That is, the condition that the orbit $u_{\sigma}(S)$ lies in $\mathcal{Q}_{\sigma}^{a}$ implies that

$$
Q_{a}\left(u_{\sigma}(S)\right) \equiv a^{2} \frac{(\sigma-u)^{2}}{(1+u)^{2}} \leq S \leq \operatorname{Min}\left\{1, h\left(u_{\sigma}(S)\right\},\right.
$$

holds all along the orbit, where

$$
h(u)=\frac{(\sigma-u)(1-3 u)}{6 u(1+u)} \leq \frac{(\sigma-u)(1-3 u)}{6 u} \leq \frac{\sigma-u}{6 u},
$$

and we can take, c.f. (6.33),

$$
a^{2}=\frac{1}{3 \sigma} .
$$

Now using (7.11) in the lower bound in (7.9) leads to

$$
\frac{\sigma-u}{1+3 u} \leq\left(\frac{1+u}{1+3 u}\right) \sqrt{3 \sigma} \sqrt{S} \leq \sqrt{3 \sigma} \sqrt{S},
$$

and applying this in (7.8) gives

$$
r_{0} \leq r_{*} e^{\frac{2 \sqrt{3 \sigma}}{1+\sigma} \sqrt{S_{0}}} .
$$

We now apply the upper bound in (7.9). Note first that

$$
\operatorname{Min}\left\{1, h\left(u_{\sigma}(S)\right\} \leq \operatorname{Min}\left\{1, \frac{(\sigma-u)(1-3 u)}{6 \hat{u}}\right\} \leq \operatorname{Min}\left\{1, \frac{\sigma-u}{6 \hat{u}}\right\},\right.
$$

where $\hat{u}$ is the (smallest) value of $u$ at which $h(\hat{u})=1$. (We need $\hat{u}$, the smallest value of $u$ that puts $h(u) \leq 1$, to bound the factor $u$ in the denominator of $h(u)$.) We can estimate $\hat{u}$ as follows. First, note that by $(7.10), h(\hat{u})=1$ is equivalent to

$$
\hat{u}=\frac{\sigma}{7+3 \sigma}-\frac{3 \hat{u}^{2}}{7+3 \sigma} \leq \frac{\sigma}{7+3 \sigma},
$$

so using the latter inequality to estimate the second term after the equality, we obtain 


$$
\begin{aligned}
\hat{u} & \geq \frac{\sigma}{7+3 \sigma}-\frac{3}{7+3 \sigma}\left(\frac{\sigma}{7+3 \sigma}\right)^{2} \\
& \geq \frac{1-1 / 7^{2}}{8} \sigma \geq \frac{\sigma}{9} .
\end{aligned}
$$

Using (7.15) in the second inequality in (7.14), (i.e., ignoring for the moment the factor $(1-3 u)$ in the middle term of $(7.14))$, implies that

$$
S \leq \operatorname{Min}\left\{1, \frac{\sigma-u}{6 \hat{u}}\right\} \leq \operatorname{Min}\left\{1, \frac{3}{2} \frac{\sigma-u}{\sigma}\right\},
$$

which implies that

$$
\sigma-u \geq \frac{2}{3} \sigma S
$$

holds all along the orbit $u=u_{\sigma}(S)$. Using (7.17) in (7.8) gives the inequality

$$
\bar{r}_{0} \geq r_{*} e^{\frac{1}{4} \sigma S_{0}} .
$$

In the case $\sigma=1 / 3$, we can improve the estimate (7.18) by using the first inequality in (7.14) to obtain

$$
S \leq \operatorname{Min}\left\{1, \frac{(\sigma-u)^{2}}{18 \hat{u}}\right\} \leq \operatorname{Min}\left\{1, \frac{1}{2} \frac{(\sigma-u)^{2}}{\sigma}\right\},
$$

and

$$
\sigma-u \geq \sqrt{2 \sigma S},
$$

in place of (7.16) and (7.17). Using (7.20) in (7.8) gives the improved inequality valid for $\sigma=1 / 3$,

$$
\bar{r}_{0} \geq r_{*} e^{\frac{\sqrt{6}}{4} \sqrt{S_{0}}} .
$$

Putting (7.13) and (7.18) together, we obtain the following bounds for the shock position $r_{0}=\bar{r}_{0}$ in terms of the initial position $r_{*}$ that apply for $0<\sigma \leq 1 / 3$ :

$$
r_{*} e^{\frac{1}{4} \sigma S_{0}} \leq r_{0} \leq r_{*} e^{\frac{2 \sqrt{3 \sigma}}{1+\sigma} \sqrt{S_{0}}} .
$$

In the case $p=\frac{1}{3} \rho$, we obtain the improved bounds

$$
r_{*} e^{\frac{\sqrt{6}}{4} \sqrt{S_{0}}} \leq r_{0} \leq r_{*} e^{\frac{3}{2} \sqrt{S_{0}}} .
$$

The following Corollary follows immediately from (7.22) and (7.23) in the case $S_{0}=1$ : 
Corollary 2. Let $\bar{r}=\bar{r}_{\text {crit }}$ denote the FRW shock position at the instant $S=1$ when the shock wave emerges from the Black Hole. Then for $0 \leq \sigma \leq 1 / 3$ we have

$$
r_{*} e^{\frac{1}{4} \sigma} \leq \bar{r}_{c r i t} \leq r_{*} e^{\frac{2 \sqrt{3 \sigma}}{1+\sigma}}
$$

while if $\sigma=1 / 3$ we have,

$$
r_{*} e^{\frac{\sqrt{6}}{4}} \leq \bar{r}_{c r i t} \leq r_{*} e^{\frac{3}{2}}
$$

where $r_{*}$ is the FRW radial coordinate of the shock wave at the instant of the Big Bang.

Note that because

$$
S=\frac{1}{N}=\frac{1}{H^{2} \bar{r}^{2}}
$$

multiplying (7.22) through by $H_{0}$ gives

$$
\frac{e^{-\frac{2 \sqrt{3 \sigma}}{1+\sigma} \sqrt{S_{0}}}}{H_{0} r_{*}} \leq \sqrt{S_{0}} \leq \frac{e^{-\frac{1}{4} \sigma S_{0}}}{H_{0} r_{*}} \leq \frac{1}{H_{0} r_{*}}
$$

and so we could use (7.27) in (7.22), (7.23) to obtain estimates involving $H_{0}$ in place of $S_{0}$. In particular we have

$$
r_{*} \leq r_{0} \leq r_{*} e^{\frac{2 \sqrt{3 \sigma}}{(1+\sigma) H_{0} r_{*}}}
$$

which reproduces the OS result $r_{0}=r_{*}$ in the limit $\sigma \rightarrow 0$.

The final theorem gives an estimate for the number of Hubble lengths to the shock wave at the instant when it first becomes visible at the FRW center, as well as an estimate for the time it takes the shock wave to emerge from the Black Hole after it first becomes visible at the FRW center.

TheOREM 9. Let $r_{*}=\lim _{S \rightarrow 0} r_{\sigma}(S)$ denote the FRW position of the shock wave at the instant of the Big Bang, and assume $0<\sigma \leq 1 / 3$. Then the shock wave will first become visible at the center $\bar{r}=0$ of the FRW spacetime at FRW time $t=t_{0}$, at the moment when the Hubble constant $H_{0}=H\left(t_{0}\right)$ satisfies

$$
H_{0} r_{*}=\frac{2}{1+3 \sigma}
$$

(assuming $R=1$ at $t=t_{0}$ ), and, the number of Hubble lengths $\sqrt{N_{0}}$ from the FRW center to the shock wave at time $t=t_{0}$ satisfies

$$
1 \leq \frac{2}{1+3 \sigma} \leq \sqrt{N_{0}} \leq \frac{2}{1+3 \sigma} e^{\sqrt{3 \sigma}\left(\frac{1+3 \sigma}{1+\sigma}\right)} .
$$

Furthermore, the time $t_{\text {crit }}>t_{0}$ at which the shock wave will emerge from the Black Hole given that it first becomes visible at $t=t_{0}$ is estimated by 


$$
\frac{2}{1+3 \sigma} e^{\frac{1}{4} \sigma} \leq \frac{t_{\text {crit }}}{t_{0}} \leq \frac{2}{1+3 \sigma} e^{\frac{2 \sqrt{3 \sigma}}{1+\sigma}},
$$

and by the better estimate

$$
e^{\frac{\sqrt{6}}{4}} \leq \frac{t_{c r i t}}{t_{0}} \leq e^{\frac{3}{2}}
$$

in the case $\sigma=1 / 3$.

Note that, for example, (7.30), (7.31) imply that at the OS limit $\sigma=0$,

$$
\sqrt{N_{0}}=2, \quad \frac{t_{c r i t}}{t_{0}}=2,
$$

and in the limit $\sigma=1 / 3$,

$$
1.8 \leq \frac{t_{\text {crit }}}{t_{0}} \leq 4.5, \quad 1<\sqrt{N_{0}} \leq 4.5,
$$

which verifies (1.2). Note also that (7.30) and (7.31) imply that the shock wave will still lie beyond one Hubble length at the time $t=t_{0}$ when it first becomes visible at the FRW center.

Proof of Theorem 9. Equation (2.41) implies that if the shock wave is first visible at $t=t_{0}, R_{0}=1$, then

$$
r_{*}=r_{\infty}=\frac{2}{(1+3 \sigma) H_{0}},
$$

which implies (7.29). To verify (7.30), multiply equation (7.28) through by $H_{0}$, and use (7.29) and (5.17).

To verify (7.31) and (7.32), let $\bar{r}_{\text {crit }}=r_{\text {crit }} R_{\text {crit }}$ denote the shock position at $S=N=1$, (the instant when the shock wave emerges from the Black Hole), and use (2.39) and (7.26) to write

$$
\frac{t_{c r i t}}{t_{0}}=\frac{H_{0}}{H_{\text {crit }}}=H_{0} \bar{r}_{\text {crit }} .
$$

Then multiplying (7.24) and (7.25) through by $H_{0}$ and using (7.33) in (7.34) gives (7.31) and (7.32), respectively. This completes the proof of Theorem 9.

8. Concluding Remarks. We have constructed global exact solutions of the Einstein equations in which the expanding FRW universe extends out to a shock wave that lies arbitrarily far beyond the Hubble length. The critical OS solution inside the Black Hole is obtained in the limit of zero pressure, but the shock wave solutions have qualitative differences from the OS solution. For example, the shock surface $\bar{r}(t)$ tends to zero as $t \rightarrow 0$, and the mass function at the shock $M(\bar{r}(t))$ is finite for all $t>0$, but unlike the OS solution, in the shock wave solution, $M(\bar{r}(t))$ tends to infinity as $t \rightarrow 0$. That is, the mass function is infinite at the instant of the Big Bang, but immediately becomes a finite decreasing function of FRW time, for all future times $t>0$. Moreover, when $p \neq 0$, the directional orientation of the shock wave motion 
relative to the various observers is determined by the entropy condition-the entropy condition chooses the explosion over the implosion. For the entropy condition we take the condition that the pressure and density be larger behind the shock wave; that is, larger on the side that receives the mass flux. This condition implies that the shock is compressive, and is sufficient to rule out expansion shocks in classical gas dynamics, $[12]$.

One can ask the question, what is the solution like beyond the shock wave at any fixed instant of time inside the Black Hole? The answer is that the TOV energy density $\bar{\rho}(\bar{r})$ and the TOV mass $M(\bar{r})$ as well, are both constant at each fixed "time" in the TOV metric beyond the shock wave, because $\bar{r}$ is the timelike TOV coordinate inside the Black Hole. This is no contradiction because what we identify (via shock matching) as the total mass function on the TOV side of the shock comes from the $d \bar{r}^{2}$ component of the metric, which is timelike inside the Black Hole. However, this TOV "total mass" matches the FRW total mass continuously at the shock surface, and the FRW total mass has the physical interpretation as $M=\frac{4 \pi}{3} \rho \bar{r}^{3}$, the integral of the energy density at each fixed time $t$ in the FRW coordinates $(t, r) .{ }^{15}$ Thus the evolution of the "total mass"is interesting and surprising inside the Black Hole.

Throughout its expansion, the strength of the shock is on the order of the energy density $\rho$ on the FRW side of the shock. At the moment when the shock wave lies at the critical distance of exactly one Hubble length from the FRW center, the TOV density and pressure are zero, and thus we argue that if the FRW density is small as well, then the shock wave continues, (with small errors), to a zero pressure OS interface leaving the Black Hole at that instant. Thus the OS solution provides the large time asymptotics of these shock wave models. That is, the interface that marks the boundary of the FRW expansion continues out through the White Hole event horizon of an ambient Schwarzschild metric at the instant when the shock wave is exactly one Hubble length from the FRW center $\bar{r}=0$, and it then continues on out to infinity along a geodesic of this Schwarzschild metric outside the Black Hole. These solutions thus indicate a scenario for the Big Bang in which the expanding universe emerges from an explosion emanating from the White Hole singularity inside the event horizon of an asumptotically flat Schwarzschild spacetime of finite mass. The model does not require the physically implausible assumption that the uniformly expanding portion of the universe is of infinite mass and extent at every fixed time, and it has the nice feature that it embeds the Big Bang singularity of cosmology within a larger spacetime, the Schwarzschild spacetime. Moreover, the model also allows for arbitrarily large densities to exist over arbitrary numbers of Hubble lengths early on in the Big Bang, a prerequisite for the standard physics of the Big Bang at early times.

One might ask how an observer near the FRW center would first detect evidence of such a cosmic shock wave. Since the shock wave emerges from the Big Bang beyond the Hubble length, the model would imply a uniform expansion throughout a region that is initially well beyond the light cone of an observer positioned near the FRW center. If the shock wave were initially far enough out, then the uncoupling of matter

\footnotetext{
${ }^{15}$ To see how $M$ can be constant on the TOV side when it measures a total mass on the FRW side, consider shock matching in $(t, \bar{r})$ coordinates. Then since the FRW mass $M$ depends only on $t$, while the TOV mass depends only on $\bar{r}$, one of them depends on the timelike coordinate and one on the spacelike coordinate in the $(t, \bar{r})$ coordinates at the shock. Thus, in the Einstein equations, the nonzero $M$ derivative ends up in a different equation on each side of the shock, giving the physical total mass on the FRW side. But on the TOV side, the derivative $M^{\prime}$ is equated to the pressure, and thus doesn't have the same interpretation as an integral of the energy density.
} 
from radiation at about 300, 000 years after the Big Bang would produce an extended region with a uniform background radiation field. This would persist until roughly the time when the Hubble length catches up to the shock wave, a time determined by the initial conditions. The influence of the solution beyond the shock wave would propagate into this radiation field at the speed of light, first appearing to an observer that is off center on the FRW side of the shock as a disturbance in the background radiation field at a point in the sky in the direction nearest the shock wave, and the disturbance would grow from that time onward.

These exact shock wave solutions give the global dynamics of strong gravitational fields in an exact solution, the dynamics is qualitatively different from the dynamics of solutions when the pressure $p \equiv 0$, and the solution suggests a Big Bang cosmological model in which the expanding universe is bounded throughout its expansion. Surprisingly, unlike shock matching outside the Black Hole, the equation of state $p=\frac{1}{3} \rho$ of early Big Bang physics, plays a special role in the equations, and for this equation of state alone, the behavior of the shock wave at the instant of the Big Bang is distinguished. But these solutions are only rough qualitative models because the equation of state on the TOV side is determined by the equations, and therefore cannot be imposed. That is, the TOV density $\bar{\rho}$ and pressure $\bar{p}$ only satisfy the loose physical bounds $0<\bar{p}<\bar{\rho}$; and on the FRW side, the equation of state is taken to be $p=\sigma \rho$, $\sigma \equiv$ const., $0<\sigma<1$. We take these bounds as implying that the equations of state are qualitatively reasonable. The entropy condition, $\rho>\bar{\rho}, p>\bar{p}$, (that the density and pressure be larger on the side that receives the mass flux), implies that the shock wave is compressive, and this fixes a time orientation for solutions, and determines a unique solution. ${ }^{15}$ However, we expect that these solutions will capture the gross dynamics arising when more general equations of state are imposed. In fact, we suggest that the global dynamics described in these solutions could only be discovered within a class of exact solutions in which simplifying assumptions are made. For more general equations of state, other waves, (e.g. rarefaction waves), would need to be present to meet the conservation constraint, and thereby mediate the transition across the shock wave. Such transitional waves would be pretty much impossible to model in an exact solution.

Finally, we note that because Einstein's theory by itself does not choose an orientation for time, it follows that if we believe that a Black Hole can exist in the forward time collapse of a mass through an event horizon as $t \rightarrow \infty$, (the time $t$ as observed in the far field), then we must also allow for the possibility of the time reversal of this process, a White Hole explosion of matter through an event horizon coming from $t \rightarrow-\infty$. These solutions might be relevant in explaining astropysical systems, such as galaxies and stellar associations [2], whose expansions appear so great as to have emerged from an event horizon at earlier times-an impossibility if one only allows the time orientation of a collapsing Black Hole, and not its time reversal, the expanding White Hole. Of course, this naturally leads one to wonder if there is a connection between the mass that mysteriously disappears into Black Hole singularities, and the mass that mysteriously emerges from White Hold singularities.

\footnotetext{
${ }^{15}$ The time orientation of a solution must be selected based on an extra condition, such as an entropy condition for shocks, because the Einstein equations and the compressible Euler equations, taken by themselves, are both time reversible, $[7,12]$. Thus the entropy condition for the shock is what determines the time orientation for the global dynamics of the solutions we construct: the FRW metric expanding outward behind a shock wave emanating from a White Hole is entropy satisfying, while its time reversal, the FRW metric contracting into a Black hole, is entropy violating. We find it interesting that the entropy condition determines a unique solution in the large.
} 


\section{REFERENCES}

[1] S.K. Blau And A.H. Guth, Inflationary cosmology. In: Three Hundred Years of Gravitation, ed. by S.W. Hawking and W. Israel, Cambridge University Press, pp. 524-603 (1987).

[2] G. Burbidge, F. Hoyle and J.V Narlikar, A Different Approach to Cosmology, Physics Today, April 1999, pp. 38-46.

[3] A. Einstein, Der feldgleichungen der gravitation, Preuss. Akad. Wiss., Berlin, Sitzber, pp. 844-847 (1915b).

[4] J. GLimm, Solutions in the large for nonlinear hyperbolic systems of equations, Comm. Pure Appl. Math., 18 (1965), pp. 697-715.

[5] S.W. Hawking and G.F.R. Ellis, The Large Scale Structure of Spacetime, Cambridge University Press, 1973.

[6] W. IsRaeL, Singular hypersurfaces and thin shells in General Relativity, IL Nuovo Cimento, Vol. XLIV B, No. 1, pp. 1-14 (1966).

[7] P.D. LAx, Hyperbolic systems of conservation laws, II, Comm. Pure Appl. Math., 10 (1957), pp. $537-566$.

[8] P.D. Lax, Shock-waves and entropy. In: Contributions to Nonlinear Functional Analysis, ed. by E. Zarantonello, Academic Press, pp. 603-634 (1971).

[9] C. Misner, K. Thorne, and J. Wheeler, Gravitation, Freeman, 1973.

[10] J.R. Oppenheimer and J.R. Snyder, On continued gravitational contraction, Phys. Rev., 56 (1939), pp. 455-459.

[11] P.J.E. PeEBles, Principles of Physical Cosmology, Princeton University Press, 1993

[12] J. Smoller, Shock-Waves and Reaction-Diffusion Equations, Springer Verlag, 1983.

[13] J. Smoller AND B. Temple, Shock-wave solutions of the Einstein equations: The Oppenheimer-Snyder model of gravitational collapse extended to the case of non-zero pressure, Arch. Rat. Mech. Anal., 128 (1994), pp. 249-297.

[14] J. Smoller And B. Temple, Astrophysical shock-wave solutions of the Einstein equations, Phys. Rev. D, 51:6 (1995), pp. 2733-2743.

[15] J. Smoller And B. Temple, Shock-wave solutions of the Einstein equations that extend the Oppenheimer-Snyder model, Arch. Rat. Mech. Anal., 138 (1997), pp. 239-277.

[16] J. Smoller And B. Temple, Shock-waves near the Schwarzschild radius and the stability limit for stars, Phys. Rev. D, 55 (1997), pp. 7518-7528.

[17] J. Smoller And B. Temple, Shock-wave solutions in closed form and the Oppenheimer-Snyder limit in general relativity, SIAM J. Appl. Math, 58:1 (1998), pp. 15-33.

[18] J. Smoller and B. Temple, On the Oppenheimer-Volkov equations in general relativity, Arch. Rat. Mech. Anal., 142 (1998), pp. 177-191.

[19] J. Smoller And B. Temple, Solutions of the Oppenheimer-Volkoff equations inside 9/8'ths of the Schwarzschild radius, Commun. Math. Phys., 184 (1997), pp. 597-617.

[20] J. Smoller And B. Temple, Shock-waves near the Schwarzschild radius and the stability limit for stars, Physical Review D, 55:12 (1997), pp. 7518-7528.

[21] J. Smoller And B. Temple, Cosmology with a shock-wave, Comm. Math. Phys., 210 (2000), pp. 275-308.

[22] J. Smoller and B. Temple, Shock-wave solutions of the Einstein equations: A general theory with examples, (to appear), Proceedings of European Union Research Network's 3rd Annual Summerschool, Lambrecht (Pfalz) Germany, May 16-22, (1999)

[23] R.M. WALD, General Relativity, University of Chicago Press, 1984.

[24] S. Weinberg, Gravitation and Cosmology: Principles and Applications of the General Theory of Relativity, John Wiley \& Sons, New York, 1972. 